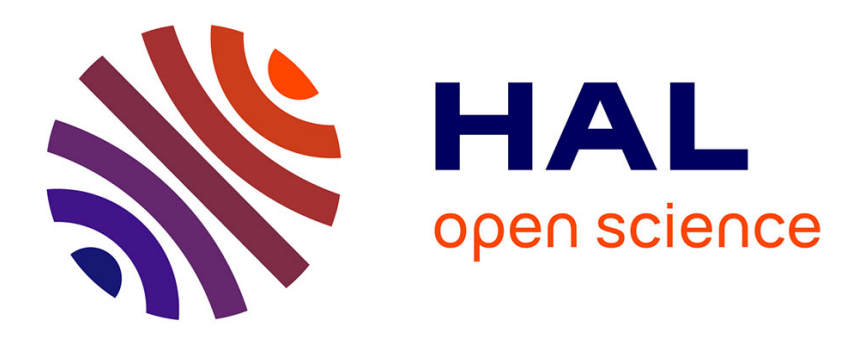

\title{
The evolution of sex along an environmental gradient
} Louise Fouqueau, Denis Roze

\section{To cite this version:}

Louise Fouqueau, Denis Roze. The evolution of sex along an environmental gradient. Evolution International Journal of Organic Evolution, In press, 10.5281/zenodo.4643637) . hal-03185708

\section{HAL Id: hal-03185708 https: / hal.sorbonne-universite.fr/hal-03185708}

Submitted on 30 Mar 2021

HAL is a multi-disciplinary open access archive for the deposit and dissemination of scientific research documents, whether they are published or not. The documents may come from teaching and research institutions in France or abroad, or from public or private research centers.
L'archive ouverte pluridisciplinaire HAL, est destinée au dépôt et à la diffusion de documents scientifiques de niveau recherche, publiés ou non, émanant des établissements d'enseignement et de recherche français ou étrangers, des laboratoires publics ou privés. 
The evolution of sex along an environmental gradient

Louise Fouqueau* ${ }^{* \dagger}$ and Denis Roze ${ }^{*, \dagger}$

* CNRS, IRL 3614 Evolutionary Biology and Ecology of Algae, 29688 Roscoff, France

† Sorbonne Université, Station Biologique de Roscoff, 29688 Roscoff, France 
Running title: Spatial heterogeneity and sex

Address for correspondence:

Denis Roze

Station Biologique de Roscoff, Place Georges Teissier, CS90074, 29688 Roscoff Cedex, France

Phone: (+33) 2564521 39, Fax: (+33) 298292324

email: roze@sb-roscoff.fr

Author contributions: LF and DR developed the simulation program, analyzed the results and wrote the article.

Acknowledgements: we thank Myriam Valero, Nick Barton and two anonymous reviewers for helpful discussions and comments, the bioinformatics and computing service of Roscoff's Biological Station (Abims platform) for computing time, the Agence Nationale de la Recherche (project SexChange, ANR-14-CE02-0001), the European Community (project MARFOR Biodiversa/004/2015) and Region Bretagne (ARED 2017 REEALG) for funding, and the Ecole Doctorale "Science de la Nature et de l'Homme, Ecologie et Evolution" for a Transhumance mobility grant to LF.

Data availability: The code for the $\mathrm{C}++$ simulation program is available on Zenodo (DOI 10.5281/zenodo.4643637). 


\begin{abstract}
While temporally changing environments generally favor sex and recombination, the effects of spatial environmental heterogeneity have been less explored. In this paper, we use a classical model of adaptation along an environmental gradient to study the selective forces acting on reproductive mode evolution in the central and marginal parts of the distribution range of a species. The model considers a polygenic trait under stabilizing selection (the optimal trait value changing across space) and includes a demographic component imposing range limits. The results show that in the central part of the range (where populations are well adapted), recombination tends to increase the mean fitness of offspring in regimes where drift is sufficiently strong (generating a benefit for sex), while it has the opposite effect when the effect of drift stays negligible. However, these effects remain weak and are easily overwhelmed by slight intrinsic fitness differences between sexuals and asexuals. In agreement with previous results, asexuality may be favored in marginal populations, as it can preserve adaptation to extreme conditions. However, a substantial advantage of asexuality is possible only in conditions maintaining a strong maladaptation of sexuals at range limits (high effective environmental gradient, weak selection at loci coding for the trait).
\end{abstract}

Keywords: Evolutionary quantitative genetics, geographic parthenogenesis, linkage disequilibrium, range margins, recombination 


\section{INTRODUCTION}

Despite the many costs associated with sexual reproduction, exclusive asexuality is rare in the eukaryotic kingdom, at least among multicellular plants and animals: the proportion of obligate asexuals is thought to be of the order of $0.1 \%$ in animals, and less than $1 \%$ in angiosperms (Vrijenhoek, 1998; Whitton et al., 2008). Interestingly, asexual lineages often present different geographical distributions than their closest sexual relatives, a phenomenon described as "geographic parthenogenesis" by Vandel (1928). In a number of cases, asexuals are found at higher latitudes or altitudes, or in environments qualified as extreme or marginal (Glesener and Tilman, 1978; Lynch, 1984; Bierzychudek, 1985; Haag and Ebert, 2004; Hörandl, 2009). A large variety of patterns are observed, however, asexuals sometimes occupying a much wider geographic range than their sexual counterparts, possibly coexisting with them in part of the range (e.g., van Dijk, 2003; Schmit et al., 2013). Several hypotheses have been proposed to explain these different distributions (Haag and Ebert, 2004; Tilquin and Kokko, 2016). Some are based on a direct selective advantage of uniparental reproduction, such as a better colonizing ability (Baker, 1955; Cuellar, 1994). Other involve spatial variation in the indirect benefits associated with sexual recombination, due to environmental heterogeneity: in particular, sex may be less advantageous in areas facing high rates of maladapted gene flow (Antonovics, 1968; Peck et al., 1998), or where inbreeding is high due to frequent population bottlenecks (Haag and Ebert, 2004), or where parasites or predators are less abundant (Glesener and Tilman, 1978). Although

these theories have hardly ever been tested empirically, the different distributions of sexual and asexual species represent an interesting avenue for exploring the possible 
benefits of sexual reproduction (Bell, 1982; Tilquin and Kokko, 2016).

Most theories on the evolutionary advantage of sex and recombination assume spatially homogeneous environments (Agrawal, 2006; Otto, 2009). Several models showed that recombination may be favoured because it increases genetic variation and therefore the rate of response to directional selection, whenever negative genetic associations (linkage disequilibria) exist within populations (that is, when alleles increasing fitness tend to be associated with alleles decreasing fitness at other loci). Such negative associations may be generated by negative epistasis (a negative curvature of the fitness function, occurring in particular in most models of optimizing selection on quantitative traits, e.g., Maynard Smith, 1980, 1988; Charlesworth, 1993; Barton, 1995; Kondrashov and Yampolsky, 1996; Vanhoenacker et al., 2018). In that case, recombination tends to decrease the mean fitness of offspring in the short term, although it increases the rate of adaptation in a longer term (Barton, 1995; Otto and Michalakis, 1998; Otto and Lenormand, 2002). Negative associations are also generated by stochastic effects occurring in finite populations (Hill and Robertson, 1966; Felsenstein, 1974), favoring sex and recombination in the absence of epistasis (e.g., Otto and Barton, 1997, 2001; Barton and Otto, 2005; Keightley and Otto, 2006; Roze, 2014).

In spatially heterogeneous environments, another possible source of linkage disequilibrium corresponds to the mixing between populations with different allele frequencies — for instance, due to spatial differences in selection (Barton and Gale, 1993). In particular, if alleles $A$ and $B$ at two loci are more frequent in a given population while alleles $a$ and $b$ are more frequent in another, dispersal will tend to produce an excess of $A B, a b$ combinations in both populations (relative to $A b, a B$ combinations). The effects of spatial heterogeneity on selection for recombination have been explored 
by Pylkov et al. (1998) and Lenormand and Otto (2000) (see also Charlesworth and Charlesworth, 1979), using models representing discrete demes connected by dispersal. They showed that, with spatial differences in selection, recombination may increase the mean fitness of offspring (for example in the simple scenario mentioned above, if $A b$, $a B$ genotypes are fitter on average than $A B, a b$ genotypes in both environments). As a consequence, recombination may be favored under wider ranges of parameters than in the absence of spatial heterogeneity, in particular when the strength of selection differs among demes (Lenormand and Otto, 2000; Otto and Lenormand, 2002; Otto, 2009; see Agrawal, 2009 for similar results on the benefits of segregation in diploids). These models have lead to very important insights, but are not ideally designed to explore the spread of asexual mutants in sexual populations living along an environmental continuum, as they generally assume recombination modifier loci with small effects and consider either a two-deme population or the island model of population structure.

Models of continuous populations living along environmental gradients have been used to explore the causes of species' range limits (Kirkpatrick and Barton, 1997; Barton, 2001; Bridle et al., 2010; Polechová and Barton, 2015; Polechová, 2018). The general framework is based on models representing the evolution of a polygenic, quantitative trait whose optimal value changes across space (Felsenstein, 1977; Slatkin, 1978; Barton, 1999), to which a demographic component is added. The most commonly used scenario considers a one-dimensional habitat, with Gaussian stabilizing selection around a phenotypic optimum whose value changes linearly across space. In the case where all parameters (except the phenotypic optimum) stay constant over space, and neglecting the effects of drift and mutation, one can show that the loci coding for the 
trait should be at linkage equilibrium: stabilizing selection tends to produce negative genetic associations, whereas migration generates positive associations (as each location receives an excess of alleles increasing the trait from one direction, and an excess of alleles decreasing the trait from the other), and the two effects compensate exactly (Felsenstein, 1977; Slatkin, 1978; Polechová and Barton, 2015). Therefore, one would expect that, in the absence of linkage disequilibrium, recombination should become neutral in this type of model. However, Felsenstein's result strictly holds in the case of an infinite, linear gradient, Gaussian stabilizing selection and no drift or mutation, and it is unclear how it may be affected by deviations from these idealized conditions. In particular, selective pressures acting on recombination may differ at the range margins, due to the asymmetric gene flow coming from central parts of the population and to stronger drift effects, preventing adaptation to marginal conditions. Under the assumptions of the infinitesimal model of quantitative trait variation, Peck et al. (1998) showed that asexual reproduction may be favored at the margins, since asexuality may maintain adaptation to extreme conditions in the face of a maladapted gene flow: this corresponds to one of the proposed hypotheses to explain geographic parthenogenesis. Ecological, demographic and genetic parameters influencing adaptation at range limits (e.g., slope of the environmental gradient, dispersal distance of individuals, strength of selection relative to drift at loci involved in local adaptation) should affect the benefit of asexuals at range margins, but this has not been formally explored.

In this paper, we extend previous models of quantitative trait evolution along environmental gradients to explore how environmental heterogeneity may affect reproductive mode evolution in central and marginal parts of the distribution range of a species. We first consider the cases of fully sexual and fully asexual populations, 
in order to explore how the reproductive mode of organisms may affect the level of adaptation and components of genetic variance maintained within populations at equilibrium. In a second step, we explore how the different parameters of the model affect the spread of asexual mutants occurring in sexual populations. The results show that either sexual or asexual reproduction may be favored in the central part of the range depending on parameter values; however, the strength of indirect selection stays weak and is quickly overwhelmed by any direct fitness effect associated with the reproductive mode. By contrast, maladaptation at range margins tends to favor the local spread of asexual clones with fitter genotypes. This benefit of asexuals is stronger for parameter values leading to an important migration load at range limits (in particular, when the effect of drift is important at loci coding for the trait), and can maintain a pattern of geographical parthenogenesis when other indirect or direct forces maintain sex in the central part of the range. However, our results show that in this case, asexuals are generally restricted to small areas at the range margins.

\section{METHODS}

General framework. Our multilocus individual-based simulation program (written in C++, and available from Zenodo) is based on Polechová and Barton's (2015) model of adaptation along an environmental gradient, to which variability in reproductive mode is added. We provide in this section a general description of the program (whose main parameters are summarized in Table 1), while more details can be found in the Supplementary Material. The model represents a population inhabiting a onedimensional habitat consisting of $n_{\mathrm{d}}$ demes connected by dispersal. Individuals are 
haploid and hermaphrodite; generations are discrete. Each generation, the number of female gametes produced by an individual is sampled from a Poisson distribution with parameter $W=e^{r}$, where $r$ represents the Malthusian fitness of the individual and is the sum of two components: a density-dependent term $r_{N}=r_{\mathrm{m}}\left(1-\frac{N}{K}\right)$, where $N$ is the size of the local deme, $K$ its carrying capacity and $r_{\mathrm{m}}$ the maximum per capita growth rate, and a local adaptation component $r_{z}=-(z-\theta)^{2} /\left(2 V_{\mathrm{s}}\right)$, where $z$ represents the value of a phenotypic trait of the individual, $\theta$ the optimal value of the trait and $V_{\mathrm{s}}$ the strength of stabilizing selection $\left(r=r_{N}+r_{z}\right)$. The trait $z$ is coded by a finite number $L$ of biallelic loci with two possible alleles (denoted 0 and 1), allele 1 increasing the value of the trait by an amount $\alpha$ : the trait value is given by $z=\alpha \sum_{i=1}^{L} X_{i}$, where $X_{i}$ equals 1 if the individual carries allele 1 at locus $i$, and 0 otherwise. Mutation occurs at a rate $u$ per generation between the two alleles at each locus (in both directions); the overall mutation rate on the trait is thus $U=u L$. The optimal trait value increases linearly across space: $\theta=b j+\theta_{0}$, where $j$ is the deme number (from 1 to $n_{\mathrm{d}}$ ) and $b$ the slope of the environmental gradient, while $\theta_{0}$ is set so that the optimal trait value is $\alpha L / 2$ in the mid-point of the 1 D-habitat (and is thus achieved in individuals carrying allele 1 at half of the loci coding for the trait). Individuals are haploid and produce gametes by mitosis; female gametes are fertilized by male gametes produced by individuals from the same deme, the contribution of each individual to the pool of male gametes being proportional to its fitness $W$ (densitydependence and local adaptation thus affect the overall reproductive output of each individual, through both male and female components). Gamete fusion occurs at random, including the possibility of selfing. Fertilization is immediately followed by meiosis to produce haploid juveniles (one per diploid zygote); we assume that the $L$ 
loci coding for the trait are evenly spaced along a linear genome with map length $R$ (average number of cross-overs at meiosis). Finally, juveniles disperse to neighboring demes according to a Gaussian dispersal kernel (discretized and truncated as explained in Appendix 3 of Polechová and Barton, 2005), where $\sigma^{2}$ measures the variance of the distance between parent and offspring (individuals move to demes 1 or $n_{\mathrm{d}}$ if their dispersal distance would lead them to move beyond the metapopulation). We also considered a different life cycle in which dispersal occurs at the gametic stage, the different events thus occurring in the order selection $\rightarrow$ dispersal $\rightarrow$ recombination (rather than selection $\rightarrow$ recombination $\rightarrow$ dispersal), but both models yielded very similar results.

Previous results and parameterization. Some of the main predictions of the model in the case of a sexual population can be found in Barton (2001) and Polechová and Barton (2015), and will be only briefly summarized here. When the environmental gradient is not too steep and genetic drift is not too strong (relative to selection) at loci coding for the trait, the population can adapt to the gradient and increase its range. In that case, a succession of clines form along space at loci coding for the trait, so that the mean population phenotype $\bar{z}$ matches the optimal phenotype in all locations. At each locus, selection pushes towards the fixation of the most common allele with a strength $s=\alpha^{2} /\left(2 V_{\mathrm{s}}\right)$ while dispersal tends to homogenize allele frequencies between demes, the balance between these two forces maintaining clines of width $4 \sigma / \sqrt{2 s}$ (Barton, 1999, 2001). Neglecting the effects of drift and mutation, one predicts that the different loci coding for the trait should be at linkage equilibrium (as the effect of stabilizing selection generating negative associations exactly compensates the effect of dispersal 
generating positive associations, e.g., Felsenstein, 1977; Polechová and Barton, 2015), and that the genetic variance $V_{\mathrm{g}}$ (the variance of $z$ ) equilibrates to $V_{\mathrm{g}}=b \sigma \sqrt{V_{\mathrm{s}}}$ in all places. This genetic variance corresponds to a "migration load" (reduction in mean fitness caused by maladapted immigrants) reducing the equilibrium deme size, given by:

$$
N_{\mathrm{eq}}=K\left(1-\frac{V_{\mathrm{g}}}{2 r_{\mathrm{m}} V_{\mathrm{s}}}\right)=K\left(1-\frac{b \sigma}{2 r_{\mathrm{m}} \sqrt{V_{\mathrm{s}}}}\right)
$$

When the parameters stay constant over space, the population may expand indefinitely, as long as genetic variation is available (Barton, 2001). A range limit may be generated if the effective environmental gradient steepens (in particular, if $b$ or $\sigma$ increase), or if the strength of drift relative to selection increases (in particular, if $K$ or $r_{\mathrm{m}}$ decrease, Polechová and Barton, 2015). In our model, we generate range limits by imposing a decrease in carrying capacity $K$ at both extremities of the metapopulation: $K$ stays equal to $K_{\max }$ in the central part, and decreases linearly towards zero on both sides, over a distance corresponding to $\omega$ demes (see Figure 1). This allows us to compare evolutionary patterns in the central part of the metapopulation (where $K=$ $\left.K_{\max }\right)$ and in the margins (areas where $K$ declines), in which gene flow is asymmetric (from more densely to less densely populated demes). We also used a model in which the range limits were generated by decreasing the per capita growth rate $r_{\mathrm{m}}$, but the results obtained were similar and will not be shown here.

The main parameters of the model are the slope of the environmental gradient $b$, the strength of stabilizing selection on the trait $V_{\mathrm{s}}$, the strength of selection acting at each locus $s$ when $\bar{z}=\theta$ (the effect of allelic change $\alpha$ being set to $\sqrt{2 V_{\mathrm{s}} s}$ ), the variance of the distance between parent and offspring $\sigma^{2}$ and the carrying capacity in the central part of the population $K_{\max }$ (together with $\omega$ that determines the slope of $K$ at the 
margins). Some of the other parameters are expected not to affect the evolutionary outcome, at least as long as they are sufficiently large: this is the case in particular for the total number of demes $n_{\mathrm{d}}$, as long as it is large enough so that the amount of genetic variation maintained in the central part of the population is not affected by edge effects. For this, the program ensures that the overall range (number of demes) of the population is always at least 3 cline widths (see Supplementary Methods), while the number of demes in the central part (with $K=K_{\max }$ ) is at least 100. The program also adjusts the total number of loci affecting the trait $(L)$ so that genotypes coding for the optimal trait value are possible in all demes. Because the average strength of linkage disequilibria is expected to depend on the harmonic mean recombination rate $\rho_{\mathrm{h}}$ between pairs of loci (e.g., Turelli and Barton, 1990; Charlesworth, 1993), the genome map length $R$ is adjusted in order to ensure a given value of $\rho_{\mathrm{h}}$ (generally 0.35) using equation A1 in Roze and Blanckaert (2014). Initialization is done as in Polechová and Barton (2015): the population initially occupies the central zone (where $\left.K=K_{\max }\right)$ and is well adapted in this area; it is then allowed to expand until it reaches its range limits (see Supplementary Material for more details).

Components of the genetic variance and migration load. We first considered separately the cases of obligately sexual and obligately asexual populations. Asexual populations follow the same life cycle as described above, except that haploid adults produce new individuals mitotically (apomixis). In both cases, the program records the mean trait value $\bar{z}$ and genetic variance $V_{\mathrm{g}}$ in all demes. The genetic variance can 
be decomposed into two terms:

$$
V_{\mathrm{g}}=\alpha^{2} \sum_{i=1}^{L} p_{i} q_{i}+\alpha^{2} \sum_{i \neq j} D_{i j}
$$

(e.g., Lynch and Walsh, 1998), where the first term depends on the frequencies of alleles 1 and 0 at each locus $i$ (denoted $p_{i}$ and $q_{i}$ ) and corresponds to the "genic" variance (denoted $V_{\mathrm{g}, 0}$ thereafter), while the second (denoted $D$ thereafter) corresponds to the overall effect of linkage disequilibria between loci $\left(D_{i j}\right)$ on the variance. Allele frequencies and $V_{\mathrm{g}}$ are measured at the different steps of the life cycle in the different demes, in order to quantify $V_{\mathrm{g}, 0}$ and $D$ under the two different reproductive modes.

The genetic load corresponds to the reduction in the mean fitness of a population due to the presence of suboptimal genotypes, and is classically defined as $L=1-\bar{W} / W_{\max }$, where $\bar{W}$ is the mean fitness and $W_{\max }$ the fitness of an optimal genotype (e.g., Crow, 1970). In our model, $L$ is generated by recurrent mutation creating new genotypes (mutation load) and by the immigration of maladapted individuals from other demes (migration load); however, the contribution of mutation should stay negligible as long as $U$ is small, and the migration load may thus be measured as:

$$
L_{\mathrm{m}}=1-\mathrm{E}\left[\exp \left(-\frac{(z-\theta)^{2}}{2 V_{\mathrm{s}}}\right)\right]
$$

where E stands for the average over all individuals in a deme. When selection is weak $\left(V_{\mathrm{g}} \ll V_{\mathrm{s}}\right)$, the average in equation 3 is approximately equal to $\exp \left[-V_{\mathrm{g}} /\left(2 V_{\mathrm{s}}\right)\right]$ $\times \exp \left[-(\bar{z}-\theta)^{2} /\left(2 V_{\mathrm{s}}\right)\right]$, showing that the load may be decomposed into two components: the effect of genetic variance $V_{\mathrm{g}}$, and the effect of displacements of the mean phenotype from the optimum (e.g., Lande and Shannon, 1996). The migration load and its two components were measured in sexual and asexual populations at equilibrium, contrasting central and marginal populations. 
In a second step, we considered the case of an initially sexual population, in which asexual mutants occur at a rate $\mu$ per generation (fixed to $10^{-3}$; the program also includes back mutations restoring sex, also at a rate $\mu$ - see Supplementary Methods). A direct cost (or benefit) of sex is introduced by multiplying the number of offspring produced by asexual females by a parameter $c: c=1$ corresponds to the situation where sexuals and asexuals have the same baseline fitness (no cost of sex), while asexual mutants have a direct advantage when $c>1$, and a disadvantage when $c<1$. We assume that asexuals do not produce any male gamete (no contagious asexuality), and only produce offspring by apomixis. As for the load, the change in the frequency of sexuals (denoted $p_{\text {sex }}$ ) due to indirect selection can be decomposed into two terms, representing the effects of differences in genetic variance $V_{\mathrm{g}}$ and differences in mean phenotype $\bar{z}$ between sexuals and asexuals (sometimes called the "short-term" and "long-term" effect of recombination, e.g., Charlesworth, 1993; Barton, 1995; Otto and Michalakis, 1998; Agrawal, 2006; Vanhoenacker et al., 2018). Based on previous works (Barton, 1995; Vanhoenacker et al., 2018), these two components can be quantified as:

$$
\begin{gathered}
S_{V_{\mathrm{g}}} \approx-\frac{1}{2\left(V_{\mathrm{g}}+V_{\mathrm{s}}\right)} \mathrm{E}\left[\left(X_{\mathrm{sex}}-p_{\mathrm{sex}}\right)(z-\bar{z})^{2}\right] \\
S_{\bar{z}} \approx-\frac{\bar{z}-\theta}{V_{\mathrm{g}}+V_{\mathrm{s}}} \mathrm{E}\left[\left(X_{\mathrm{sex}}-p_{\mathrm{sex}}\right)(z-\bar{z})\right]
\end{gathered}
$$

where $X_{\text {sex }}$ equals 0 and 1 in asexual and sexual individuals, respectively. The program measures the moments appearing on the right-hand sides of equations 4 and 5 in the different demes, thus providing estimates of the relative strengths of the two components of indirect selection for sex $\left(S_{V_{\mathrm{g}}}\right.$ and $\left.S_{\bar{z}}\right)$. 


\section{RESULTS}

\section{Adaptation to environmental heterogeneity in sexual and asexual popula-}

tions. We first consider the case of obligately sexual or asexual populations (without letting the reproductive mode evolve). In regimes where the population can adapt to the environmental gradient, the amount of genetic variation maintained at each location is predicted to increase with the slope of the gradient and with the dispersal distance of individuals, and to decrease with the strength of stabilizing selection (e.g., Barton, 1999). Assuming that the mean phenotype matches the optimum everywhere and that selection acting on the trait is weak $\left(V_{\mathrm{g}} \ll V_{\mathrm{s}}\right.$, so that differences in fitness between individuals remain small), the per-generation change in genetic variance due to stabilizing selection is $\Delta_{\text {sel }} V_{\mathrm{g}} \approx-V_{\mathrm{g}}^{2} / V_{\mathrm{s}}$, while the change due to dispersal is $\Delta_{\text {disp }} V_{\mathrm{g}} \approx(b \sigma)^{2}$ (Barton, 1999). If linkage disequilibria stay negligible in sexual populations (so that the effect of recombination can be neglected), and if the mutational variance $V_{\mathrm{m}}=\alpha^{2} U$ is small relative to $(b \sigma)^{2}$, the equilibrium genetic variance is given by $\Delta_{\mathrm{sel}} V_{\mathrm{g}} \approx-\Delta_{\text {disp }} V_{\mathrm{g}}$, leading to $V_{\mathrm{g}} \approx b \sigma \sqrt{V_{\mathrm{s}}}$. This shows that gene flow may substantially inflate the genetic variance, so that the hypothesis that $V_{\mathrm{g}} \ll V_{\mathrm{s}}$ may not necessarily hold. In that case, more accurate expressions can be obtained by assuming that the distribution of phenotypes in each location is approximately Gaussian: the change in $V_{\mathrm{g}}$ caused by selection then becomes $\Delta_{\text {sel }} V_{\mathrm{g}} \approx-V_{\mathrm{g}}^{2} /\left(V_{\mathrm{g}}+V_{\mathrm{s}}\right)$ (e.g., Bulmer, 1985), so that the equilibrium value of $V_{\mathrm{g}}$ after dispersal, before selection is given by:

$$
V_{\mathrm{g}, \text { aft disp }} \approx \frac{1}{2}\left[(b \sigma)^{2}+b \sigma \sqrt{(b \sigma)^{2}+4 V_{\mathrm{s}}}\right]
$$

while the variance after selection, before dispersal is:

$$
V_{\mathrm{g}, \text { aft sel }} \approx V_{\mathrm{g}, \text { aft disp }}-(b \sigma)^{2}
$$


the average between both quantities being $(b \sigma / 2) \sqrt{(b \sigma)^{2}+4 V_{\mathrm{s}}}$ (and thus converging to $b \sigma \sqrt{V_{\mathrm{s}}}$ when $(b \sigma)^{2} \ll V_{\mathrm{s}}$, so that $\left.V_{\mathrm{g}} \ll V_{\mathrm{s}}\right)$. Figure 2 shows that the predictions from equations 6 and 7 match our simulation results for all tested values of $b, \sigma$ and $V_{\mathrm{g}}$ (this was verified for both sexual and asexual populations, as well as for the selection $\rightarrow$ dispersal $\rightarrow$ recombination life cycle). The same results are obtained for different strengths of selection per locus $s$, including values such that $N_{\text {eq }} s \ll 1$ (so that the effect of drift is strong relative to selection at each locus) and $N_{\text {eq }} s \gg 1$.

Despite the fact that sexual and asexual populations display approximately the same amount of genetic variance at equilibrium, slight differences are observed. In particular, while the average variance usually matches very well the theoretical prediction in asexuals (except for high values of $\sigma$ ), sexuals may display slightly higher or lower $V_{\mathrm{g}}$ (Figure $\mathrm{S} 1$ ). These differences are due to the effect of recombination: although linkage disequilibria are small at the time when recombination occurs, they may be slightly positive or negative depending on parameter values (Figure 3). When linkage disequilibria are positive $(D>0)$, recombination tends to decrease $V_{\mathrm{g}}$ by reducing the second term of equation 2 , while recombination has the opposite effect when $D<0$. Figure $3 \mathrm{~A}$ and $3 \mathrm{~B}$ indicate that positive $D$ arises when the per locus strength of selection $s$ is small relative to the effect of drift, while $D$ becomes negative as deme size or $s$ increases. Although we could not obtain an analytical expression for $D$ at migration-selection-drift equilibrium, insights can be gained from quasi-linkage equilibrium results under weak selection: indeed, when $D$ remains small and $V_{\mathrm{g}} \ll V_{\mathrm{s}}$, the change in $D$ due to selection is approximately $-\left(V_{\mathrm{g}, 0}\right)^{2} / V_{\mathrm{s}}$ (e.g., Turelli and Barton, 1990), while the change due to dispersal is $(b \sigma)^{2}$. As long as $D$ remains small, and ignoring the effects of mutation and drift, $V_{\mathrm{g}, 0} \approx V_{\mathrm{g}} \approx b \sigma \sqrt{V_{\mathrm{s}}}$, so that the two effects 
compensate exactly (Felsenstein, 1977; Polechová and Barton, 2015). Mutation tends to increase the genic variance $V_{\mathrm{g}, 0}$, so that the effect of selection becomes stronger than the effect of dispersal, generating negative $D$ (Slatkin, 1978, Figure 3F). By contrast, drift tends to decrease $V_{\mathrm{g}, 0}$, generating positive $D$. As shown by Figure $3, \sigma$ and $V_{\mathrm{s}}$ have only limited effects on the contribution of linkage disequilibria to $V_{\mathrm{g}}$, while increasing $b$ tends to increase the relative importance of positive $D$ in the weak selection regime.

In asexuals, simulations indicate that while the genetic variance $V_{\mathrm{g}}$ remains constant over time, $D$ tends to become more and more negative (while $V_{\mathrm{g}, 0}$ becomes more and more positive), due to the constant appearance of new genotypes carrying different combinations of compensatory mutations (the same effect is predicted to occur in the case of fully selfing populations, e.g., Lande, 1977; Lande and Porcher, 2015). Although the observed $V_{\mathrm{g}}$ matches well the theoretical prediction (equation 6) when averaged over demes (Figure S1), we observed that a stable pattern of periodic oscillations of $V_{\mathrm{g}}$ and $\bar{z}-\theta$ sets up along the metapopulation for relatively low values of $s$ and/or high values of $\sigma$ (e.g., for $s=0.001$ and $\sigma=6$ in Figure 4). This corresponds to a situation in which optimal genotypes are predominant only in some demes separated by a fixed distance: in those demes, $\bar{z}=\theta$ and $V_{\mathrm{g}}$ reaches a minimal value, while the demes in between contain mixtures of maladapted immigrant genotypes, causing $V_{\mathrm{g}}$ to increase and $\bar{z}$ to deviate from $\theta$ (in this situation, $D$ is initially positive and becomes negative over time, as shown by Figure S2). As shown by Figure S3, the amplitude of these oscillations increases as deme size decreases. This clustering of the phenotypic distribution of asexuals along an environmental gradient has already been shown by Polechová and Barton (2005) using a deterministic version of the same model (see also Champagnat and Méléard, 2007), and is due to a structural instability of the smooth 
solution with constant $V_{\mathrm{g}}$ and $\bar{z}=\theta$ when the mutational variance is small (in other words, two asexual clones with very similar phenotypes cannot coexist in adjacent demes). Interestingly, in this regime the phenotypic distribution is multimodal and thus non-Gaussian in most of the demes, but the Gaussian approximation still provides a correct prediction of the average genetic variance over demes (Figures 2, S1).

As shown by Figure 4, periodic fluctuations do not occur in the case of sexual populations: $V_{\mathrm{g}}$ stays approximately constant over space, for all tested parameter values. In both sexuals and asexuals, mean phenotypes usually stay close to the optimum in the central part of the population (although some deviations arise in the oscillating regime just described), so that the migration load is mostly generated by $V_{\mathrm{g}}$. Therefore, sexuals have a slightly higher load than asexuals in the regime where $D<0$ in sexuals (recombination increases $V_{\mathrm{g}}$ ) and a slightly lower load in the regime where $D>0$ (recombination decreases $V_{\mathrm{g}}$, see Figure 5 ). However, the oscillations of $V_{\mathrm{g}}$ occurring in asexuals in the high $\sigma /$ low $s$ regime generate oscillations of the load, as shown by Figure S4.

At range margins, the lower population size causes a decrease in the genic variance $V_{\mathrm{g}, 0}$ in sexual populations. Interestingly, we generally observed that this decrease in $V_{\mathrm{g}, 0}$ is almost exactly compensated by an increase in $D$, so that $V_{\mathrm{g}}$ stays approximately constant in most of the range, and remains well predicted by equation 6 (see Figure S5 for an example). Moreover, Figure 6 shows that the mean phenotype tends to depart from the optimum at range limits (due to drift and to asymmetric gene flow from more densely populated demes), becoming an important component of the load (in particular when selection per locus $s$ is weak, as shown by Figures 6 , S6 and S7). Maladaptation at range limits is more important in the case of asexual 
populations (Figures 6, S6, S7), probably due to the increased effects of drift caused by interference between selected loci in the absence of recombination (e.g., Barton, 2010). As a consequence, the species range tends to be smaller in asexuals than in sexuals (Figure S8), the difference being again mostly marked when $s$ is small. Despite the fact that purely asexual populations tend to suffer more from migration load at range limits, we will see that asexual mutants can nevertheless be favored at the range limits of sexual populations, since they are not affected by migration load in this case.

Evolution of reproductive mode. When asexual mutants are introduced within sexual populations, we observed that in the absence of any direct fitness effect of the reproductive mode $(c=1)$, sexual reproduction stays predominant in the central part of the range for parameter values leading to a lower load in sexuals than in asexuals, while asexuality prevails for parameter values leading to a lower load in asexuals. In particular, Figure 7A shows that the frequency of sexuals $\left(p_{\text {sex }}\right)$ stays high in the central part of the range when the per locus strength of selection $s$ is small (leading to positive linkage disequilibria, Figure 3), while asexual mutants invade at higher values of $s$ (Figure S9 shows that the effects of $b, \sigma$ and $V_{\mathrm{s}}$ on the frequency of sexuals can also be predicted from their effects on the relative migration load of sexuals and asexuals at equilibrium). However, in the parameter region where sex is favored (low $s$ ), asexuals are maintained at non-negligible frequencies (e.g., $p_{\text {sex }} \approx 0.8$ for $s=0.001$ ). This may be due to the fact that differences in mean fitness between sexual and asexual populations are weak, but also to the oscillations occurring in asexual populations in the low $s$ regime (Figure 4) which may favor asexuals over sexuals in certain demes. Figure 7B confirms that selection on the reproductive mode is mainly driven by the 
difference in genetic variance $V_{\mathrm{g}}$ between sexual and asexual populations (quantified by $S_{V_{\mathrm{g}}}$, given by equation 4 ), while the mean phenotype stays close to the optimum (on average) for both reproductive modes (leading to $S_{\bar{z}} \approx 0$ ). Differences in $V_{\mathrm{g}}$ and in the migration load between sexual and asexual population stay relatively weak (Figure 5), so that any direct fitness effect of the reproductive mode quickly prevails and leads to the near fixation of sexuals when $c<1$ (direct cost of asexuality), and to the near fixation of asexuals when $c>1$ (direct cost of sex, see Figure 8).

By contrast, the evolution of reproductive mode at the range limits cannot be predicted from the migration load of purely sexual and purely asexual populations at equilibrium. Indeed, while purely asexual populations always suffer from a similar or higher load than sexual populations at range limits (Figure S6), we observed that asexual clones may often spread in peripheral populations. When sex is maintained in the central part of the range (either due to a higher load in asexuals when $c \sim 1$ or to an intrinsic advantage of sexuals, i.e., $c<1$ ), the asexual population maintained at range limits does not suffer from migration load (since immigrants coming from the central area are mostly sexual), leading to a stable pattern of geographic parthenogenesis. The spread of asexuals at range margins is due to the maladaptation of sexual populations in these areas, generating an advantage for locally adapted mutant clones: indeed, asexuals are favored because they can maintain phenotypes that are closer to the optimum $\left(S_{\bar{z}}<0\right.$, see Figure $\left.\mathrm{S} 10\right)$. This advantage of asexuals increases with the degree of maladaptation of sexuals, which (as shown by Polechová and Barton, 2015) depends on the effective environmental gradient (proportional to $b \sigma / \sqrt{V_{\mathrm{s}}}$ ) and on the effect of drift relative to selection at loci coding for the trait (see Figure 9). For sufficiently strong values of $b \sigma / \sqrt{V_{\mathrm{s}}}$ and sufficiently weak selection relative to drift at loci 
coding for the trait (low $s$ ), asexuality may thus be maintained at range limits despite an intrinsic advantage of sexuals $(c<1$, see Figures S11 - S16 showing the abundance of sexuals and asexuals along space for different parameter values). In general, the maintenance of geographic parthenogenesis in the present model is only possible for sufficiently weak selection at loci coding for the trait: under stronger selection (e.g., $s=0.05$ in Figures S15, S16), the benefit of asexuals at range limits stays weak and is easily overwhelmed by any advantage associated with sex (represented here by $c<1$ ), while such an advantage of sex is necessary to maintain sexuals in the central part.

Finally, Figures S11 - S16 show that the area in which asexuals are predominant at range limits (under conditions favoring sex in the central area) generally stays rather small. As shown by Figure 10A, decreasing the slope of the change in carrying capacity over space (by increasing $\omega$ ) has little effect on the size of this area. Although we have seen that the advantage of asexuals at range limits increases with $b \sigma / \sqrt{V_{\mathrm{s}}}$ (Figure 9 ), Figure 10B shows that asexuals tend to be confined in a smaller area (measured in units of dispersal distance $\sigma$ ) as $b \sigma / \sqrt{V_{\mathrm{s}}}$ increases. This is likely due to the fact that although a locally adapted clone may benefit from a stronger advantage when $b \sigma / \sqrt{V_{\mathrm{s}}}$ is higher (due to the stronger maladaptation of sexuals), this benefit is quickly lost as individuals move to neighboring demes (since the effective environmental gradient is stronger).

\section{DISCUSSION}

Temporal environmental change generally favors sex and recombination due to the fact that recombination increases the amount of genetic variation upon which direc- 
tional selection can act (Charlesworth, 1993; Barton, 1995; Otto and Michalakis, 1998; Otto, 2009; Becks and Agrawal, 2012). By contrast, the effect of spatial environmental change on selection for sex has been somewhat less studied. Pylkov et al. (1998) and Lenormand and Otto (2000) demonstrated that spatial variation in the direction or strength of selection may sometimes generate a short-term benefit for recombination (increase in mean fitness of recombinant offspring), while Peck et al. (1998) showed that asexuality may be favored at species range margins as it can preserve locally adapted genotypes. In this study, we used a classical model of evolution along an environmental gradient (Felsenstein, 1977; Slatkin, 1978; Barton, 1999; Polechová and Barton, 2015) to further explore the effects of spatial heterogeneity on reproductive mode evolution. We found that in situations where demes are locally adapted (i.e., when the average phenotype matches the local optimum), recombination either increases or decreases the mean fitness of offspring depending on the relative strengths of selection, mutation and drift. While the effect of stabilizing selection generating negative LD exactly compensates the effect of dispersal generating positive LD when mutation and drift are neglected (Felsenstein, 1977; Polechová and Barton, 2015), mutation tends to produce negative LD (Slatkin, 1978) while drift generates positive LD (recombination being beneficial under positive LD as it tends to increase the proportion of locally adapted genotypes, and disadvantaged under negative LD). This leads to a benefit for sexual reproduction when deme size is sufficiently small, and when selection and mutation are sufficiently weak. However, in most cases the difference in mean fitness between sexuals and asexuals stays rather weak, so that indirect selection acting on the reproductive mode is easily overwhelmed by even slight direct costs or benefits associated with asexuality. This model therefore does not represent a realistic 
scenario for the evolutionary maintenance of costly sex within populations. However, it is interesting to note that while stabilizing selection generally favors asexuality in equilibrium populations living in homogeneous habitats (in the absence of mutational bias, e.g., Charlesworth, 1993; Vanhoenacker et al., 2018), a spatial environmental gradient may erase this advantage of asexuals (at least in the central part of the species range), making it easier for sex to be favored through other mechanisms. Exploring the effects of selection, drift and dispersal on the sign and magnitude of linkage disequilibria (and the resulting strength of selection for sex and recombination) under more general scenarios (including different forms of fitness function or non-linear changes in phenotypic optimum over space) would be of interest, as they may generate stronger selection on recombination.

From an empirical perspective, a better understanding of the selective forces affecting the evolution of reproductive modes could be gained by measuring the effect of recombination on the mean and variance in fitness among offspring (e.g., Sharp and Otto, 2016). This type of data remains scarce, however (Peters and Otto, 2003; Becks and Agrawal, 2011 and references therein) and to our knowledge, only one study used fitness measures obtained under natural conditions (Kelley et al., 1988). Based on our results and those of previous models (Felsenstein, 1977; Lenormand and Otto, 2000 ), it would be of particular interest to compare the effects of recombination on offspring fitness in populations subject to different forms of environmental heterogeneity. In particular, while recombination is expected to increase the variance in fitness and decrease mean fitness under spatially homogeneous stabilizing selection, these effects may vanish or even reverse in the case of populations living along environmental gradients. This could be done in principle by comparing the fitness distributions of 
sexually and asexually produced offspring in species using both reproductive modes (Kelley et al., 1988; Becks and Agrawal, 2012), although the fact that sexually and asexually produced propagules are often not ecologically or physiologically equivalent represents a challenge for such experiments.

Our simulations of obligately asexual populations showed that in some regimes, adaptation cannot be maintained at all geographic locations (a result that was already obtained in previous numerical explorations of similar models, in particular Polechová and Barton, 2005; Champagnat and Méléard, 2007). In such situations, the population consists of different subtypes adapted to specific locations separated by a more or less constant distance (which increases with the dispersal distance of individuals). As a consequence, the genetic variance and level of adaptation fluctuate along space in a periodic fashion (Figure 4). Comparing the patterns of adaptation along environmental gradients in sexual and asexual populations represents another possible direction for empirical studies, either by comparative analyses of natural populations or by using experimental evolution approaches. At range limits, our simulations indicate that obligately asexual populations generally suffer from a stronger migration load (caused by migration bias from more densely populated habitats) than obligately sexual populations, in particular when the strength of selection per locus is small. Similarly, Vanhoenacker et al. (2018) showed that mutational biases tend to have a stronger impact on the mutation load of asexual populations, due to the reduced efficiency of directional selection in asexuals. Extending our model to include a migration bias in the central part of the population (that may be caused by winds or ocean currents in natural habitats) could thus be of interest, as it may increase the strength of selection for sex. 
Finally, our results confirm that the constant maladaptation of peripheral populations can favor the spread of asexual mutants adapted to these extreme environments, which may lead to a pattern of geographic parthenogenesis when sex is maintained in the central part of the range (either by direct or indirect selection). The strength of selection for asexuality correlates positively with the magnitude of the migration load in peripheral areas, which itself increases with the steepness of the environmental gradient $(b)$, the variance of the dispersal distance $\left(\sigma^{2}\right)$ and the strength of stabilizing selection $\left(1 / V_{\mathrm{s}}\right)$, but decreases with the strength of selection per locus $(s)$. This is consistent with the results obtained by Peck et al. (1998) using the infinitesimal model (extremely small strength of selection per locus), also showing patterns of geographic parthenogenesis in the presence of a direct fitness cost associated with asexuality. However, our results show that geographic parthenogenesis is difficult to maintain in this type of model when the strength of selection acting at loci involved in local adaptation is not sufficiently small. Furthermore, asexuality usually stays restricted to a small area at range limits where density is low. While this may be compatible with some of the reported cases of geographic parthenogenesis (although estimates of $\sigma$ are rarely available), situations in which asexuals occupy a very large area (e.g., van Dijk, 2003; Schmit et al., 2013) seem more difficult to explain with this type of hypothesis. Demographic advantages associated with uniparental reproduction (better colonizing ability, Baker, 1955) may possibly explain such patterns in the case of recent geographic expansions: such benefits of asexuality could be incorporated in the model by considering dioecious or self-incompatible sexual organisms.

While changes in species ranges and evolutionary dynamics at population expansion fronts have been the subject of increased attention in a context of accelerating 
global change (e.g., Gilbert et al., 2017; Peischl et al., 2015; Williams et al., 2019), the selective pressures acting on the evolution of recombination or reproductive systems during range expansion remain little explored. Yet, shifts in reproductive system have profound demographic and genetic consequences that should in turn affect population expansion (Barrett et al., 2008). Preliminary results obtained using our simulation models indicate that asexual mutants may spread at expansion fronts due to the important migration load maintained in these areas, considerably slowing the expansion by reducing the adaptive potential of marginal populations. This question should be further explored under more general scenarios regarding population demography and the genetic architecture of fitness. 


\section{LITERATURE CITED}

Agrawal, A. F. 2006. Evolution of sex: why do organisms shuffle their genotypes? Curr. Biol. 16:R696-R704.

. 2009. Spatial heterogeneity and the evolution of sex in diploids. Am. Nat. 174:S54-S70.

Antonovics, J. 1968. Evolution in closely adjacent plant populations V. Evolution of self-fertility. Heredity 23:219-238.

Baker, H. G. 1955. Self-compatibility and establishment after 'long-distance' dispersal. Evolution 9:347-348.

Barrett, S. C. H., R. I. Colautti, and C. G. Eckert. 2008. Plant reproductive systems and evolution during biological invasion. Mol. Ecol. 17:373-383.

Barton, N. H. 1995. A general model for the evolution of recombination. Genet. Res. $65: 123-144$.

. 1999. Clines in polygenic traits. Genet. Res. 74:223-236.

—. 2001. Adaptation at the edge of a species' range. Pp. 365-392 in J. Silvertown and J. Antonovics, eds. Integrating Ecology and Evolution in a Spatial Context. Blackwell, London.

—. 2010. Genetic linkage and natural selection. Phil. Trans. Roy. Soc. (Lond.) B $365: 2559-2569$. 
Barton, N. H. and K. S. Gale. 1993. Genetic analysis of hybrid zones. Pp. 13-45 in R. G. Harrison, ed. Hybrid zones and the evolutionary process. Oxford University Press, New York, USA.

Barton, N. H. and S. P. Otto. 2005. Evolution of recombination due to random drift. Genetics 169:2353-2370.

Becks, L. and A. F. Agrawal. 2011. The effect of sex on the mean and variance of fitness in facultatively sexual rotifers. J. Evol. Biol. 24:656-664.

. 2012. The evolution of sex is favoured during adaptation to new environments. PLoS Biology 5:e1001317.

Bell, G. 1982. The Masterpiece of Nature. University of California Press, Berkeley.

Bierzychudek, P. 1985. Patterns in plant parthenogenesis. Pp. 197-217 in S. C. Stearns, ed. The evolution of sex and its consequences. Birkhauser Verlag, Basel.

Bridle, J. R., J. Polechová, M. Kawata, and R. K. Butlin. 2010. Why is adaptation prevented at ecological margins? New insights from individual-based simulations. Ecol. Lett. 13:485-494.

Bulmer, M. G. 1985. The Mathematical Theory of Quantitative Genetics, 2nd edition. Oxford University Press, Oxford.

Champagnat, N. and S. Méléard. 2007. Invasion and adaptive evolution for individualbased spatially structured populations. J. Math. Biol. 55:147-188.

Charlesworth, B. 1993. Directional selection and the evolution of sex and recombination. Genet. Res. 61:205-224. 
Charlesworth, D. and B. Charlesworth. 1979. Selection on recombination in clines. Genetics 91:581-589.

Crow, J. F. 1970. Genetic loads and the cost of natural selection. Pp. 128-177 in K. Kojima, ed. Mathematical Topics in Population Genetics. Springer-Verlag, Berlin.

Cuellar, O. 1994. Biogeography of parthenogenetic animals. Biogeogr. 70:1-13.

Felsenstein, J. 1974. The evolutionary advantage of recombination. Genetics 78:737756.

1977. Multivariate normal genetic models with a finite number of loci. Pp. 227-245 in E. Pollak, O. Kempthorne, and T. Bailey Jr, eds. Proceedings of the International Conference on Quantitative Genetics. Iowa State Univ. Press, Ames, IA.

Gilbert, K. J., N. P. Sharp, A. L. Angert, G. L. Conte, J. A. Draghi, F. Guillaume, A. L. Hargreaves, R. Matthey-Doret, and M. C. Whitlock. 2017. Local adaptation interacts with expansion load during range expansion: maladaptation reduces expansion load. Am. Nat. 189:368-380.

Glesener, R. R. and D. Tilman. 1978. Sexuality and the components of environmental uncertainty: clues from geographic parthenogenesis in terrestrial animals. Am. Nat. 112:659-673.

Haag, C. R. and D. Ebert. 2004. A new hypothesis to explain geographic parthenogenesis. Ann. Zool. Fenn. 41:539-544.

Hill, W. G. and A. Robertson. 1966. The effect of linkage on limits to artificial selection. Genet. Res. 8:269-294. 
Hörandl, E. 2009. Geographical parthenogenesis: opportunities for asexuality. Pp. 1611869 in I. Schoen, K. Martens, and P. van Dijk, eds. Lost sex. Springer, Amsterdam.

Keightley, P. D. and S. P. Otto. 2006. Interference among deleterious mutations favours sex and recombination in finite populations. Nature 443:89-92.

Kelley, S. E., J. Antonovics, and J. Schmitt. 1988. A test of the short-term advantage of sexual reproduction. Nature 331:714-716.

Kirkpatrick, M. and N. H. Barton. 1997. Evolution of a species' range. Am. Nat. $150: 1-23$.

Kondrashov, A. S. and L. Y. Yampolsky. 1996. Evolution of amphimixis and recombination under fluctuating selection in one and many traits. Genet. Res. 68:165-173.

Lande, R. 1977. The influence of the mating system on the maintenance of genetic variability in polygenic characters. Genetics 86:485-498.

Lande, R. and E. Porcher. 2015. Maintenance of quantitative genetic variance under partial self-fertilization, with implications for the evolution of selfing. Genetics 200:891-906.

Lande, R. and S. Shannon. 1996. The role of genetic variation in adaptation and population persistence in a changing environment. Evolution 50:434-437.

Lenormand, T. and S. P. Otto. 2000. The evolution of recombination in a heterogeneous environment. Genetics 156:423-438.

Lynch, M. 1984. Destabilizing hybridization, general purpose genotypes and geographic parthenogenesis. Quart. Rev. Biol. 59:257-290. 
Lynch, M. and J. B. Walsh. 1998. Genetics and Analysis of Quantitative Traits. Sinauer Associates, Sunderland, MA.

Maynard Smith, J. 1980. Selection for recombination in a polygenic model. Genet. Res. 35:269-277.

- 1988. Selection for recombination in a polygenic model — the mechanism. Genet. Res. 51:59-63.

Otto, S. P. 2009. The evolutionary enigma of sex. Am. Nat. 174:S1-S14.

Otto, S. P. and N. H. Barton. 1997. The evolution of recombination: removing the limits to natural selection. Genetics 147:879-906.

2001. Selection for recombination in small populations. Evolution 55:19211931.

Otto, S. P. and T. Lenormand. 2002. Resolving the paradox of sex and recombination. Nat. Rev. Genet. 3:252-261.

Otto, S. P. and Y. Michalakis. 1998. The evolution of recombination in changing environments. Trends Ecol. Evol. 13:145-151.

Peck, J. R., J. M. Yearsley, and D. Waxman. 1998. Explaining the geographic distributions of sexual and asexual populations. Nature 391:889-892.

Peischl, S., M. Kirkpatrick, and L. Excoffier. 2015. Expansion load and the evolutionary dynamics of a species range. Am. Nat. 185:E81-E93.

Peters, A. D. and S. P. Otto. 2003. Liberating genetic variance through sex. BioEssays 25:533-537. 
Polechová, J. 2018. Is sky the limit? On the expansion threshold of a species' range. PLoS Biology 16:e2005372.

Polechová, J. and N. H. Barton. 2005. Speciation through competition: a critical review. Evolution 59:1194-1210.

2015. Limits to adaptation along environmental gradients. Proc. Natl. Acad. Sci. U. S. A. 112:6401-6406.

Pylkov, K. V., L. A. Zhivotovsky, and M. W. Feldman. 1998. Migration versus mutation in the evolution of recombination under multilocus selection. Genet. Res. $71: 247-256$.

Roze, D. 2014. Selection for sex in finite populations. J. Evol. Biol. 27:1304-1322.

Roze, D. and A. Blanckaert. 2014. Epistasis, pleiotropy and the mutation load in sexual and asexual populations. Evolution 68:137-149.

Schmit, O., S. N. S. Bode, A. Camacho, D. J. Horne, D. K. Lamatsch, K. Martens, M. J. F. , Martins, T. Namiotko, G. Rossetti, J. Rueda-Sevilla, I. Schön, J. Vandekerkhove, and F. Mesquita-Joanes. 2013. Linking present environment and the segregation of reproductive modes (geographical parthenogenesis) in Eucypris virens (Crustacea: Ostracoda). J. Biogeogr. 40:2396-2408.

Sharp, N. P. and S. P. Otto. 2016. Evolution of sex: using experimental genomics to select among competing theories. BioEssays 38:751-757.

Slatkin, M. 1978. Spatial patterns in the distributions of polygenic characters. J. Theor. Biol. 70:213-228. 
Tilquin, A. and H. Kokko. 2016. What does the geography of parthenogenesis teach us about sex? Phil. Trans. Roy. Soc. (Lond.) B 371:20150538.

Turelli, M. and N. H. Barton. 1990. Dynamics of polygenic characters under selection. Theor. Popul. Biol. 38:1-57.

van Dijk, P. J. 2003. Ecological and evolutionary opportunities of apomixis: insights from Taraxacum and Chondrilla. Phil. Trans. Roy. Soc. (Lond.) B 358:1113-1121.

Vandel, A. 1928. La parthénogenèse géographique. Contribution à l'étude biologique et cytologique de la parthénogenèse naturelle. Bull. Biol. France Belg. 62:164-281.

Vanhoenacker, E., L. Sandell, and D. Roze. 2018. Stabilizing selection, mutational bias, and the evolution of sex. Evolution 72:1740 -1758.

Vrijenhoek, R. C. 1998. Animal clones and diversity. BioScience 48:617-628.

Whitton, J., C. J. Sears, E. J. Baack, and S. P. Otto. 2008. The dynamic nature of apomixis in the angiosperms. Int. J. Plant Sci. 169:169-182.

Williams, J. L., R. A. Hufbauer, and T. E. Miller. 2019. How evolution modifies the variability of range expansion. Trends Ecol. Evol. 34:903-913. 


\begin{tabular}{c|c}
$b$ & Slope of the environmental gradient \\
\hline$\sigma^{2}$ & Variance of the distance between parent and offspring \\
\hline$V_{\mathrm{s}}$ & Strength of stabilizing selection \\
\hline$s$ & Strength of selection acting at each locus $\left(\frac{\alpha^{2}}{2 V_{\mathrm{s}}}\right)$ \\
\hline$K_{\max }$ & Carrying capacity in the central part of the population \\
\hline$\omega$ & Number of demes over which $K$ decreases from $K_{\text {max }}$ to zero \\
\hline$r_{\mathrm{m}}$ & Maximum per capita growth rate \\
\hline$L$ & Number of loci coding for the quantitative trait \\
\hline$n_{\mathrm{d}}$ & Number of demes \\
\hline$c$ & Cost of sex
\end{tabular}

Table 1. Main parameters of the model 


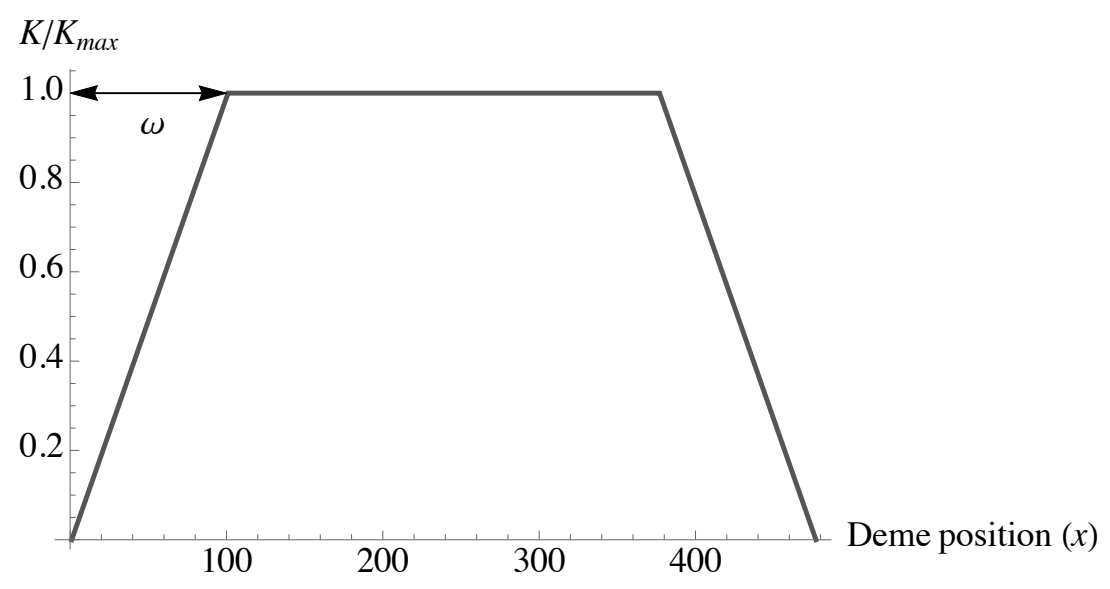

Figure 1. Variation of the carrying capacity $K$ across space: $K$ equals $K_{\max }$ in the central part of the population, and decreases linearly towards zero at both extremities (over $\omega$ demes). The number of demes in the central part depends on the values of $s$ and $\sigma$ (see Methods). 

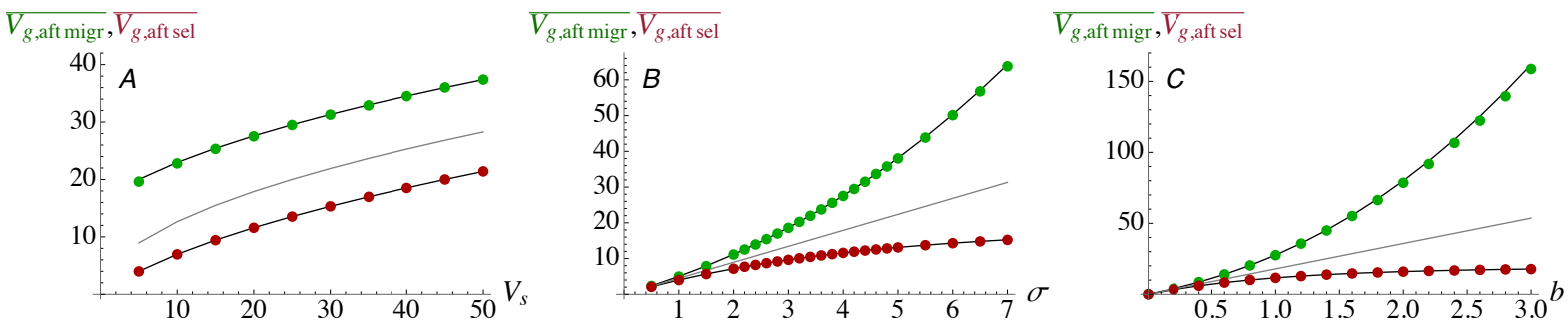

Figure 2. Average genetic variance $V_{\mathrm{g}}$ per deme after selection (red) and after migration (green) in the central part of the population, as a function of the strength of stabilizing selection $V_{\mathrm{s}}(\mathrm{A})$, the standard deviation of the distance between parent and offspring $\sigma(\mathrm{B})$ and the slope of the environmental gradient $b(\mathrm{C})$. The black curves correspond to the predictions from equations 6 and 7 , and the grey curve to $b \sigma \sqrt{V_{\mathrm{s}}}$. The dots correspond to simulation results in the case of a sexual population with $s=0.001, K_{\max }=500, \omega=100, V_{\mathrm{s}}=20$ (in B, C), $\sigma=4$ (in A, C), $b=1$ (in A, B), $r_{\mathrm{m}}=1.1$ and $U=10^{-3}$. Simulation results obtained with $s=0.05$ for the same values of the other parameters, as well as results obtained in the case of an asexual population (for both $s=0.001$ and $s=0.05$ ) are undistinguishable on the scale of the figures and are thus not shown. 

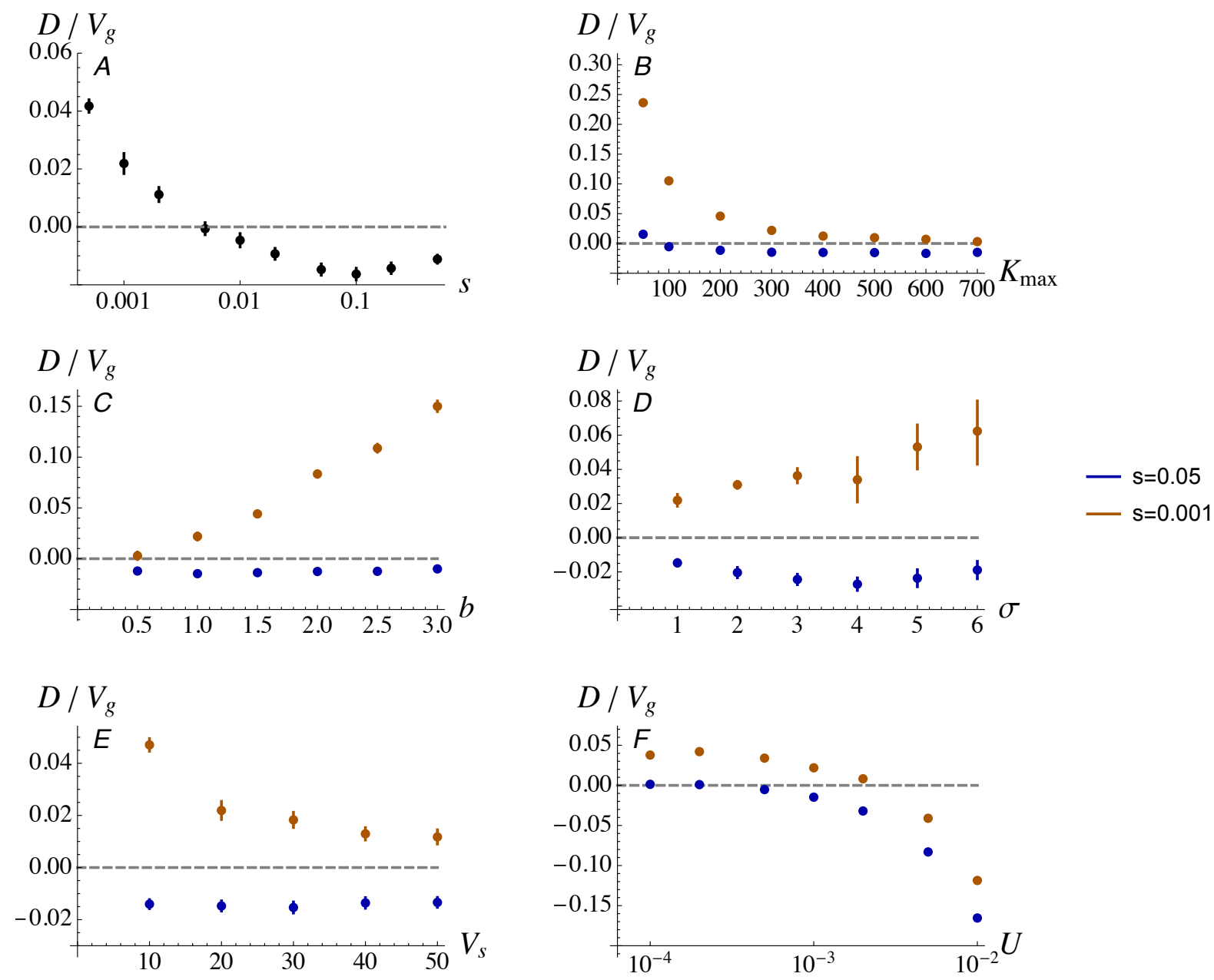

Figure 3. Contribution of linkage disequilibria to the genetic variance $D / V_{\mathrm{g}}$ (where $D$ corresponds to the second term of equation 2) at equilibrium in the central part of a sexual population, as a function of $s$ (on a log scale), $K_{\max }, b, \sigma, V_{\mathrm{s}}$ and $U$ (on a $\log$ scale). Default parameter values are $b=1, V_{\mathrm{s}}=20, \sigma=1, K_{\max }=300, \omega=100$, $r_{\mathrm{m}}=1.1$ and $U=10^{-3}$. 
$s=0.05, \sigma=1$
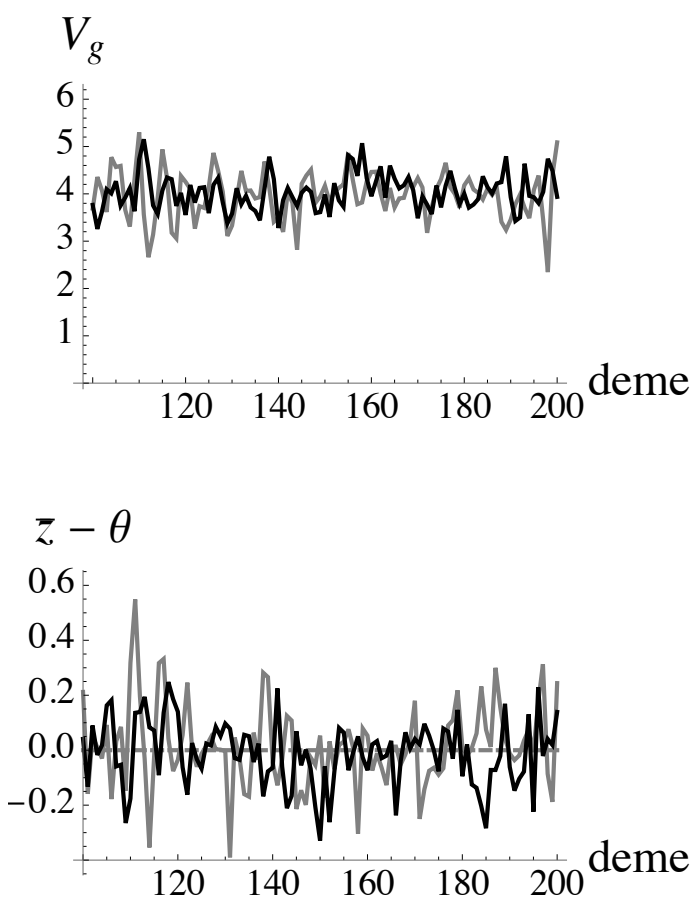

$s=0.001, \sigma=6$

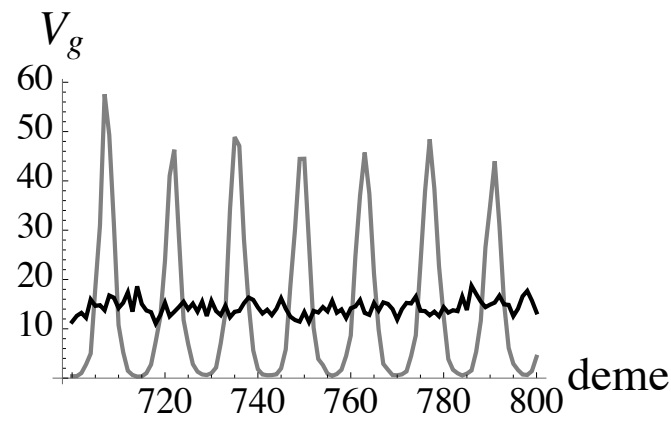

$z-\theta$

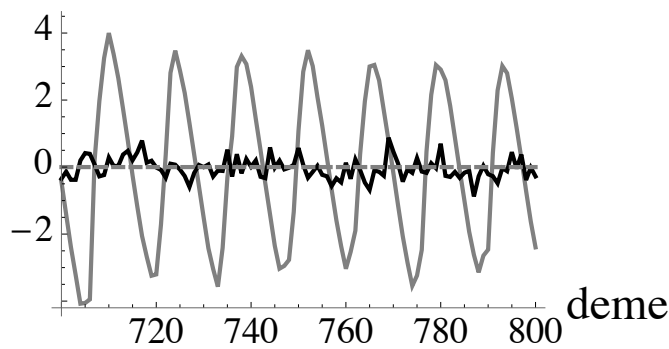

Figure 4. Genetic variance $V_{\mathrm{g}}$ and difference between the mean phenotype $\bar{z}$ and the optimum $\theta$ (measured after selection) along space (in part of the central region of the population, where $K=K_{\max }$ ) in sexuals (black) and asexuals (grey). Parameter values are $b=1, V_{\mathrm{s}}=20, K_{\max }=300, \omega=100, r_{\mathrm{m}}=1.1, U=10^{-3}$, and $s=0.05$, $\sigma=1$ (left), $s=0.001, \sigma=6$ (right). A stable pattern of periodic oscillations along space occurs in asexual populations when $\sigma$ is high and $s$ is small. 

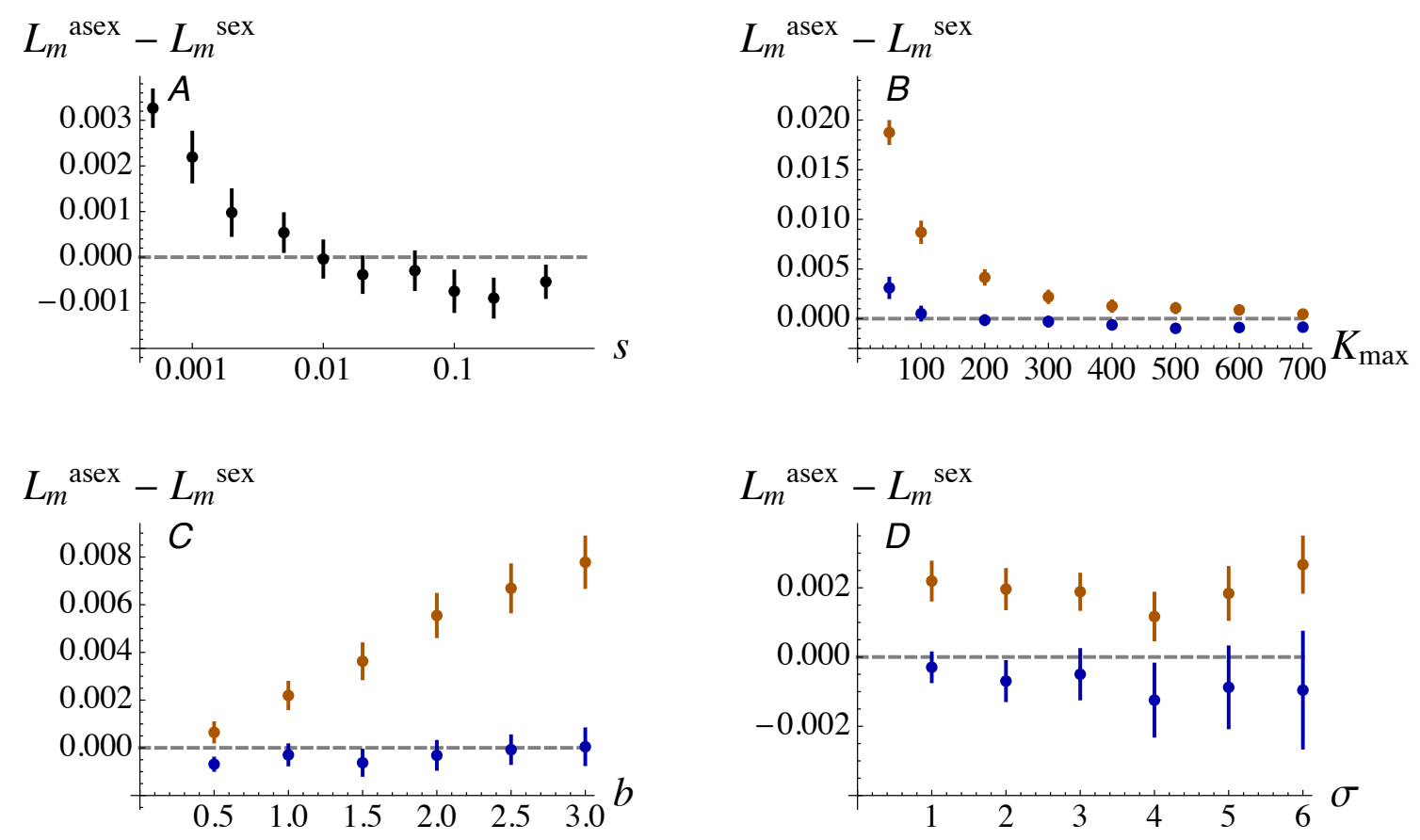

$L_{m}^{\text {asex }}-L_{m}{ }^{\text {sex }}$
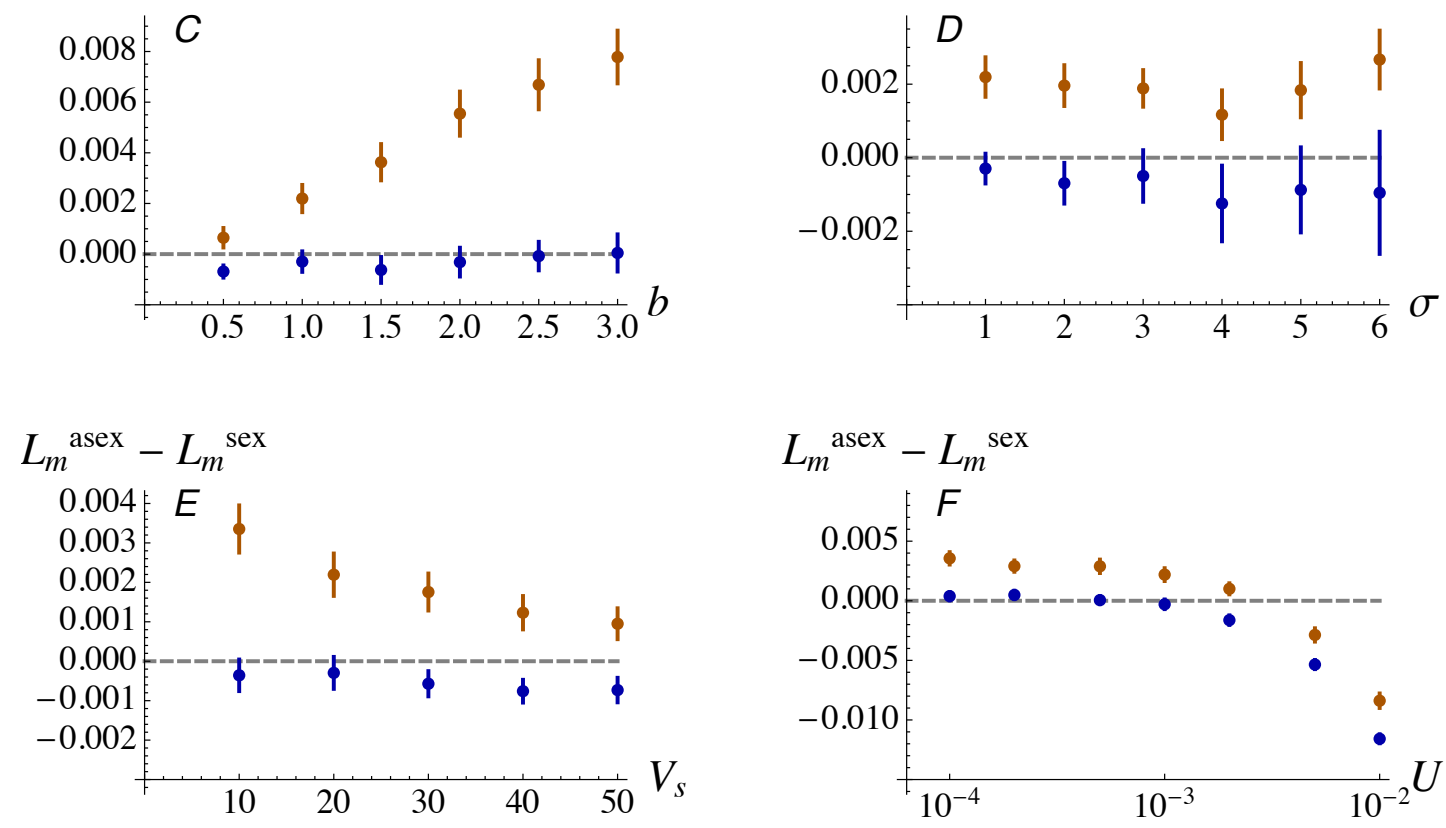

Figure 5. Difference in migration load $L_{\mathrm{m}}$ (measured by equation 3 ) between asexual and sexual populations at equilibrium, in the central part of the distribution range, for the same parameter values as in Figure 3. The parameter region in which sexuals have a lower load $\left(L_{m}{ }^{\text {asex }}-L_{m}{ }^{\text {sex }}>0\right)$ corresponds to the parameter region in which $D>0$ in sexuals (see Figure 3 ). 


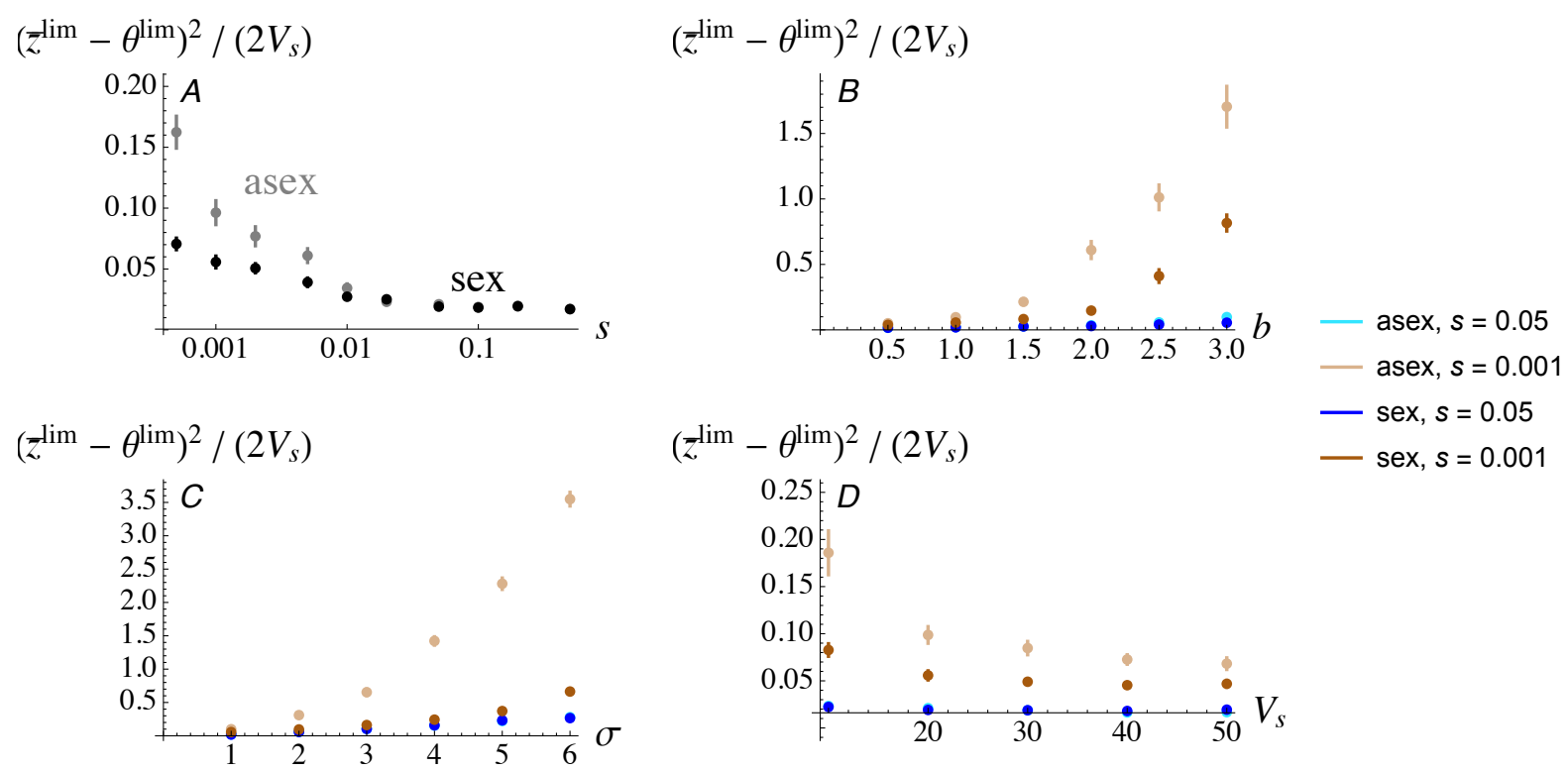

Figure 6. Squared difference between the mean phenotype and the optimum, scaled by $2 V_{\mathrm{s}}$ (corresponding to the effect of the deviation of the mean phenotype from the optimum on the migration load) at the margins of the distribution range, as a function of $s, b, \sigma$ and $V_{\mathrm{s}}$. Parameter values are the same as in Figures 3 and 5 . Values are averaged over extreme demes whose size is comprised between 10 and 50 individuals. In the top left figure, black and grey dots correspond to sexuals and asexuals, respectively. 

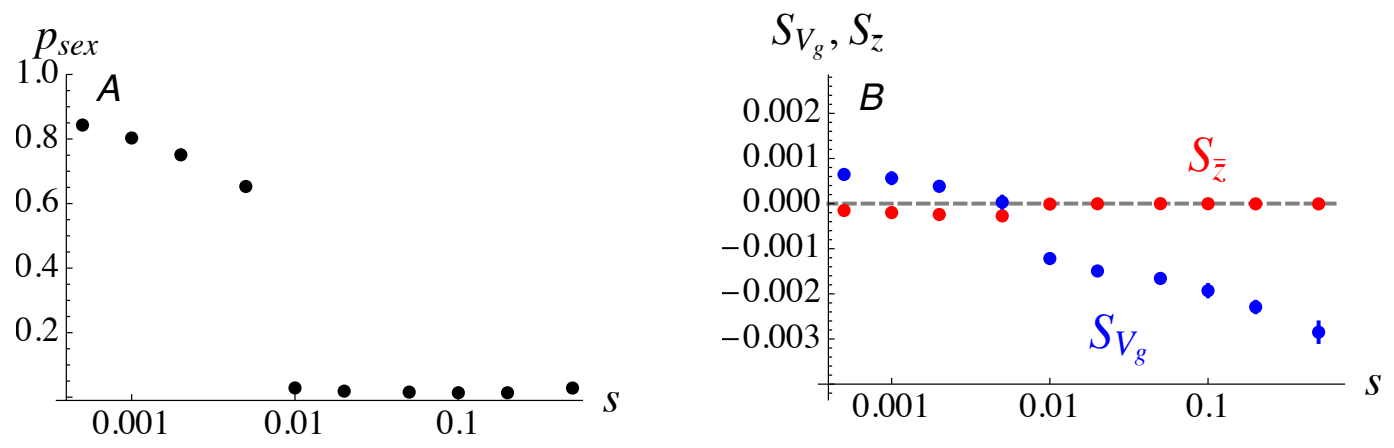

Figure 7. Left: mean frequency of sexuals at equilibrium in the central part of the distribution range as a function of the per-locus strength of selection $s$ (on log scale), for the same default parameter values as in Figure 3, and $c=1$ (no cost of sex). Right: the two components of selection for sex $S_{V_{\mathrm{g}}}$ (blue) and $S_{\bar{z}}$ (red), given by equations 4 and 5 . 


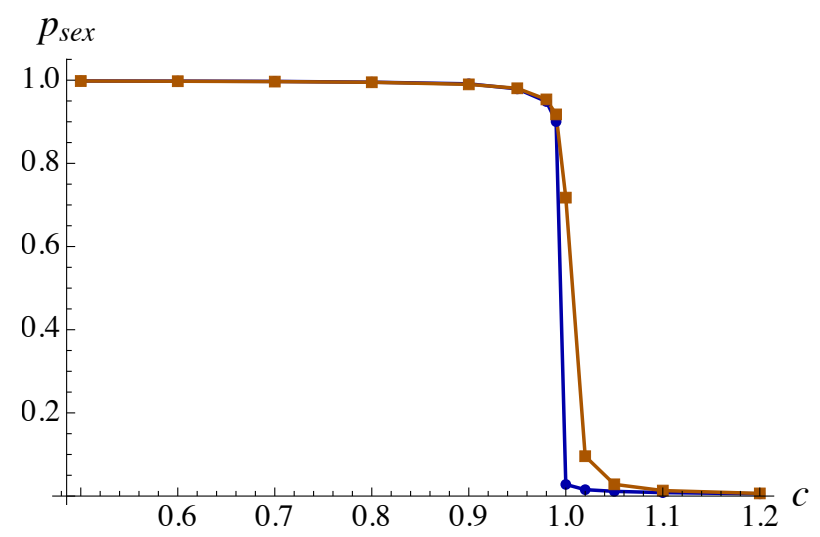

Figure 8. Mean frequency of sexuals at equilibrium in the central part of the distribution range as a function of the cost of sex $c$ under strong $(\mathrm{s}=0.05$, blue) and weak selection $(\mathrm{s}=0.001$, brown $)$. Default parameter values are as in Figure 3. 

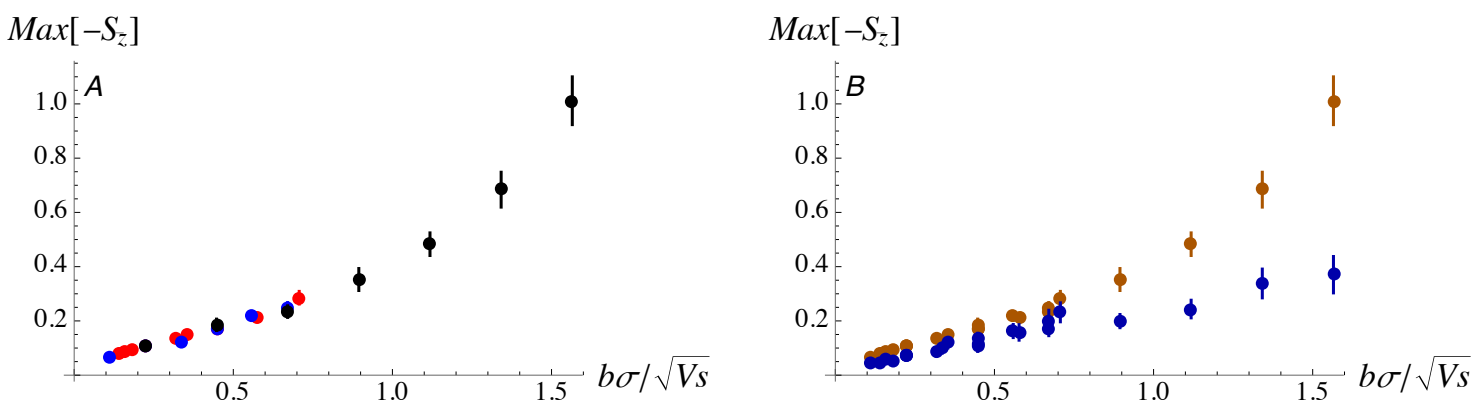

Figure 9. Maximum benefit of asexuals at range limits as a function of $b \sigma / \sqrt{V_{\mathrm{s}}}$. The maximum value of $-S_{\bar{z}}$ (given by equation 5 and representing the benefit of asexuals due to the fact that their mean phenotype is closer to the optimum) was measured over all marginal populations (areas in which the carrying capacity decreases) during the first 1000 generations after asexual mutants are introduced. Dots show averages over 30 replicate simulations. In A, default parameter values are as in Figure 3 (in particular, $\left.\sigma=1, b=1, V_{\mathrm{s}}=20\right)$ with $s=0.001$. Black dots correspond to different values of $\sigma$ (from $\sigma=1$ to $\sigma=7$ ), blue dots to different values of $b$ (from $b=0.5$ to $b=3)$ and red dots to different values of $V_{\mathrm{s}}\left(\right.$ from $V_{\mathrm{s}}=2$ to $\left.V_{\mathrm{s}}=50\right)$. Brown dots in B correspond to the same results as in A $(s=0.001)$, while blue dots correspond to equivalent results obtained for $s=0.05$ (and the same values of the other parameters). In both $\mathrm{A}$ and $\mathrm{B}$, the parameter $c$ was set to $c=0.98$ (slight intrinsic advantage of sexuals, so that sex can be maintained in the central part of the range when $s=0.05)$. 


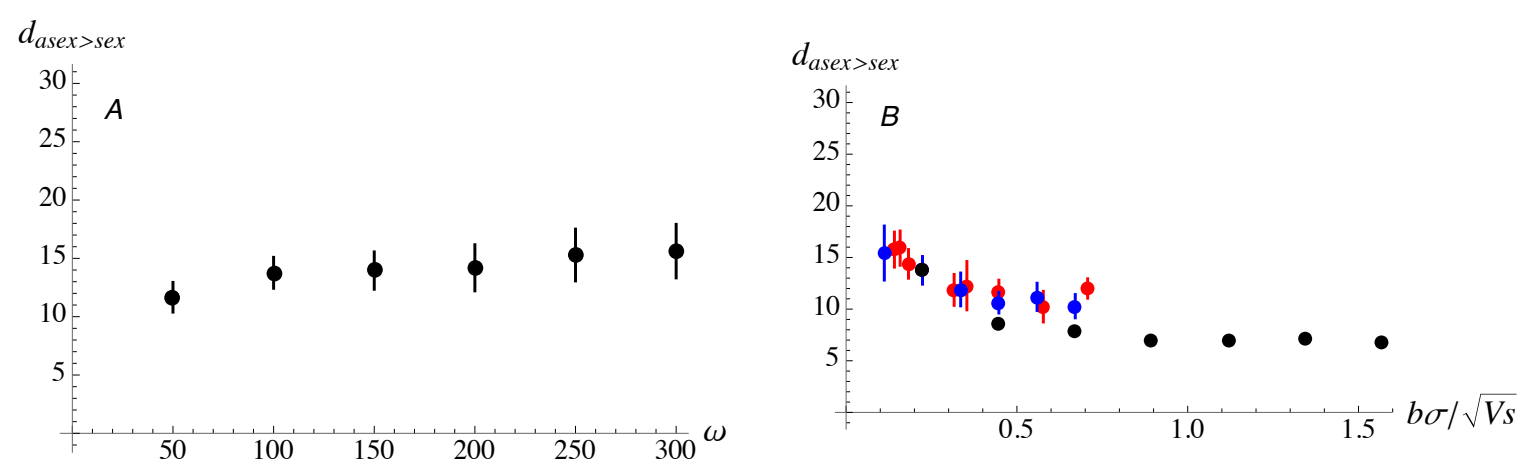

Figure 10. Scaled distance (number of demes divided by the standard deviation of the dispersal distance $\sigma$ ) over which the density of asexuals exceeds the density of sexuals at range limits. A: as a function of the number of demes over which carrying capacity decreases $(\omega$, see Figure 1$)$. B: as a function of $b \sigma / \sqrt{V_{\mathrm{s}}}$ (colors have the same meaning as in Figure 9A). Default parameter values are as in Figure 3 with $s=0.001$ and $c=1$ (no cost of sex). 


\section{SUPPLEMENTARY METHODS}

Initialization. All demes are initially populated with $N_{\text {eq }}$ individuals, given by equation $1\left(r_{\mathrm{m}}\right.$ is readjusted if needed to ensure that $N_{\text {eq }} \geq 30$ in the central part of the population). As in Polechová and Barton (2015), a series of clines is set up at loci coding for the quantitative trait in the central part of the population, in order to ensure that demes in the central part are already well adapted. At migration-selection equilibrium, the equation for each cline is given by:

$$
p(x)=\frac{1}{1+\exp \left(-\frac{4\left(x-x_{0}\right)}{\omega_{\mathrm{cl}}}\right)}
$$

where $p$ is the frequency of allele $1, x$ the spatial position and $x_{0}$ the position of the center of the cline (at which $p=0.5$ ), and where the cline width is given by $\omega_{\mathrm{cl}}=4 \sigma / \sqrt{2 s}$ (Barton 1999). The total number of demes $n_{\mathrm{d}}$ is adjusted if necessary to ensure that $n_{\mathrm{d}} \geq 3 \omega_{\mathrm{cl}}$; in the case of narrow clines, the program also ensures that the central part of the population comprises at least 100 demes. The number of loci coding for the trait $(L)$ is always at least 1000 , and is also readjusted if necessary to ensure that $L \geq b n_{\mathrm{d}} / \alpha$, so that the phenotypic optimum can be reached everywhere. The $L_{\mathrm{cl}}$ initial clines are separated by a spatial distance $\alpha / b$, and a proportion $\left(L-L_{\mathrm{cl}}\right) / 2$ of loci (randomly sampled among the $L$ loci) are fixed for allele 1 (the remaining loci being fixed for allele 0 ), in order to ensure that $\bar{z}=\theta$ in the area where clines are set up. For each of the $L_{\mathrm{cl}}$ clines, a locus is sampled randomly, and the allele carried by each individual of deme $x$ at this locus is switched to 1 if a random number drawn from a uniform distribution between 0 and 1 is lower than $p(x)$. Demes located outside the area where clines are set up are not well adapted $(\bar{z} \neq \theta)$, and as a consequence 
population density quickly drops to zero in those demes; they can be later recolonized as the population expands.

Measures and numbers of generations. Genetic and genic variances are measured every 1,000 generations within each deme, after each step of the life cycle (selection, recombination and dispersal). The genetic variance $V_{\mathrm{g}}$ corresponds to the variance of $z$ within the deme, while the genic variance is given by $V_{\mathrm{g}, 0}=\alpha^{2} \sum_{i=1}^{L} p_{i} q_{i}$, where $p_{i}$ and $q_{i}$ are the frequencies of the two alleles at locus $i$. Values of $V_{\mathrm{g}}$ and $V_{\mathrm{g}, 0}$ after selection (before recombination) are obtained by weighting each individual by its relative fitness. The program also measures the mean phenotype $\bar{z}$ and migration load, given by equation 3. Except for the simulation results shown on Figure 9, a single replicate was performed for each set of parameters, over a large enough number of generations to ensure that an equilibrium was reached long before the end of the simulation. In the case of obligately sexual populations (without letting the reproductive mode evolve), simulations generally lasted 50,000 generations, and averages were performed over the last 40,000 generations (we checked by visual inspection that equilibrium was reached during the first 10,000 generations). For most simulations of obligately asexual populations, only phenotypic moments were measured and we used a modified program in which only the total number of alleles 1 in the genome of each individual is represented (without representing explicitly the $L$ loci, in order to increase execution speed); however, measures of $V_{\mathrm{g}, 0}$ and $D$ (Figure S2) were obtained for some parameter values using the standard program. Because asexual populations took longer to reach their range limit, simulations were run over a larger number of generations $(500,000$ in the case of Figures 5, 6, S6-S8). Simulations in which we let 
the reproductive mode evolve generally lasted 90,000 generations, asexual mutants being introduced after 60,000 preliminary generations (each sexual individual becoming asexual with probability $\mu=10^{-3}$ ).

Selection - dispersal - recombination. A different program represented gametic dispersal, so that recombination occurs just after dispersal, before selection (instead of occurring after selection, before dispersal as in our standard program). In that case, female gametes migrate first according to the dispersal kernel. The probability that a female gamete present in deme $i$ is fertilized by a male gamete immigrating from deme $j$ is then given by $\overline{W_{\sigma^{\Uparrow} j}} m_{i j} / \sum_{k}\left(\overline{W_{\sigma^{\rtimes k}}} m_{i k}\right)$ where $\overline{W_{\sigma^{\Im} j}}$ is the average male fitness in deme $j$, and $m_{i j}$ the probability that a gamete produced in deme $j$ migrates to deme $i$. Once the deme of origin of the male gamete has been drawn, a father is sampled according to its male fitness as in the standard program. The results obtained using this modified program were very similar to those obtained under the selection recombination - dispersal life cycle (not shown). 


\section{SUPPLEMENTARY FIGURES}
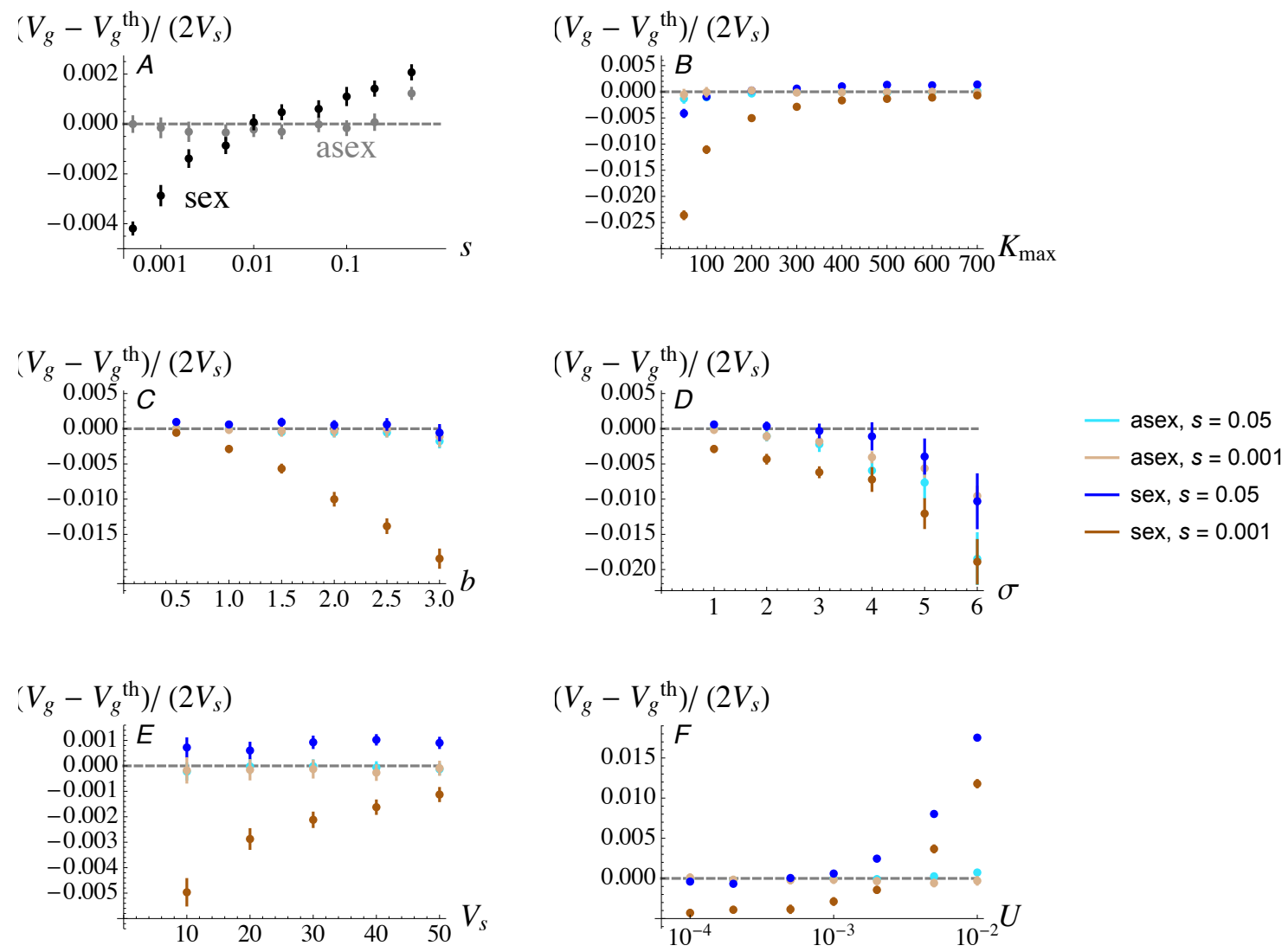

Figure S1. Difference between the average genetic variance $V_{g}$ per deme observed in the simulations (in the central part of the population) and the theoretical prediction given by equation $6\left(V_{g}^{\text {th }}\right)$, scaled by $2 V_{\mathrm{s}}$, in sexual and asexual populations. Parameter values are as in Figure 3. 


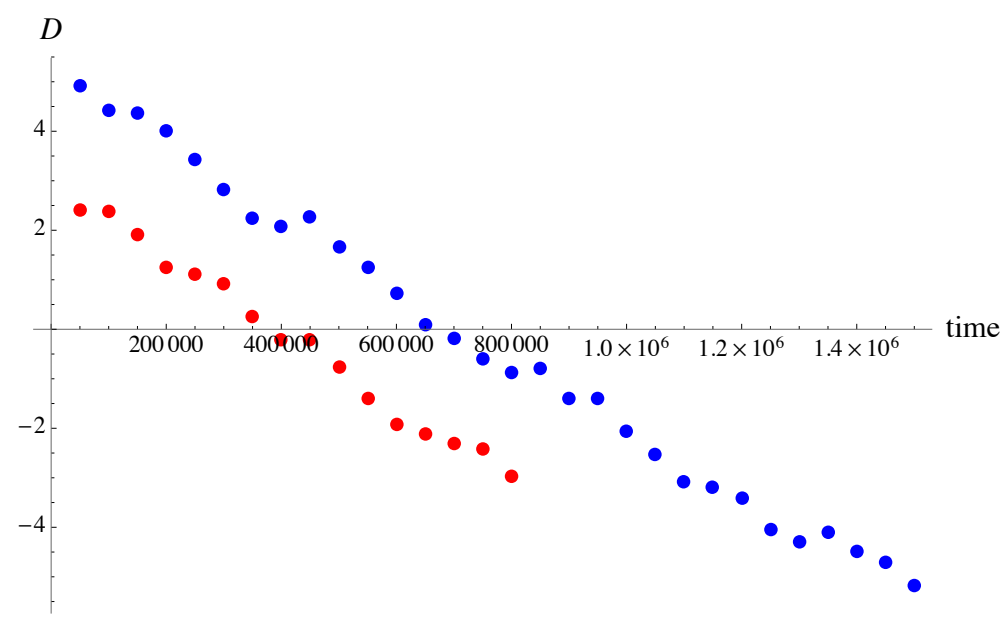

Figure S2. Dynamics of the contribution of linkage disequilibria to the genetic variance ( $D$, averaged over central demes) over time, in asexual populations with $s=0.001$, $V_{\mathrm{s}}=20, \sigma=4, b=1, r_{\mathrm{m}}=1.1, K_{\max }=300$ (blue) and $K_{\max }=3,000$ (red). 


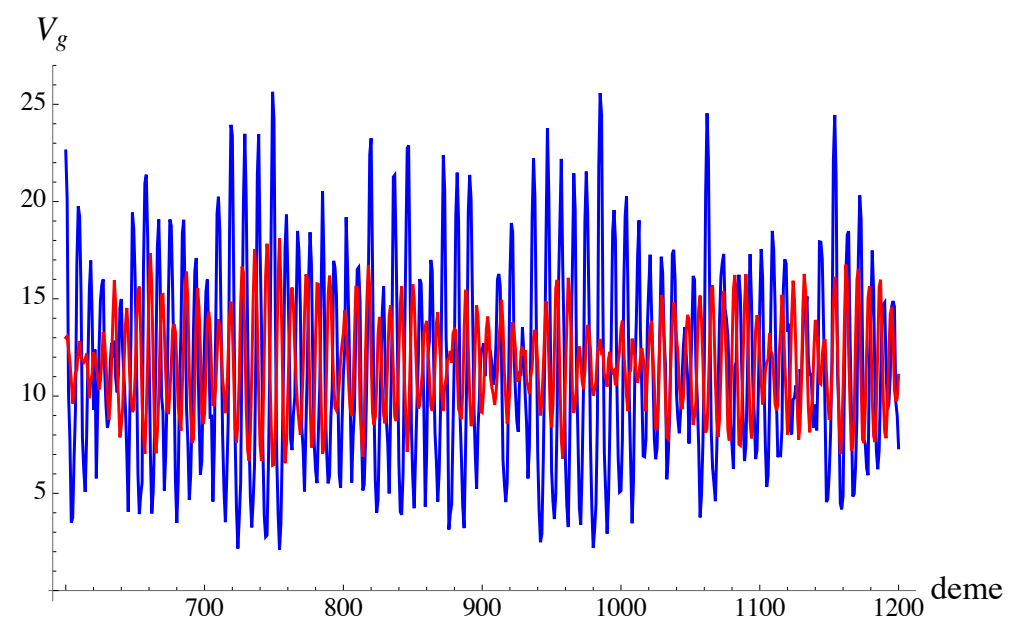

Figure S3. Fluctuations of the genetic variance $V_{\mathrm{g}}$ over space in asexual populations, at generation 500,000 (same parameter values and colors as in Figure S2). 


$$
\mathrm{s}=0.05, \sigma=1
$$

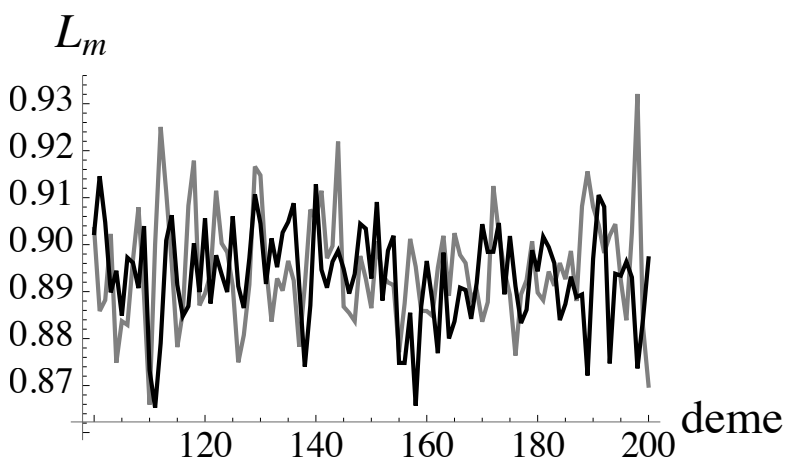

$\mathrm{s}=0.001, \sigma=6$

$$
L_{m}
$$

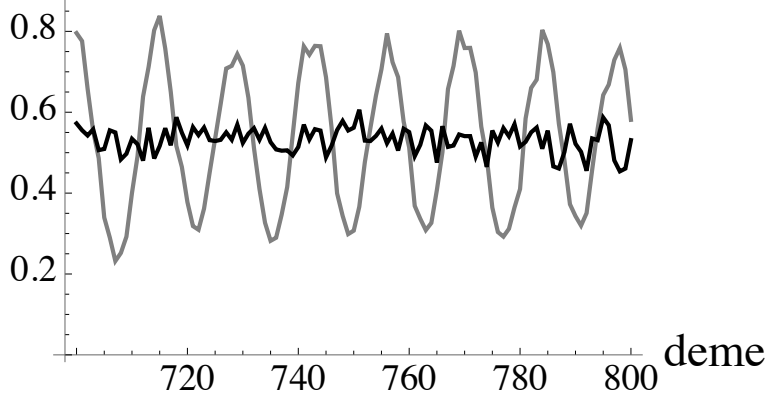

Figure S4. Variation of the migration load along space in sexuals (black) and asexuals (grey), for the same parameter values as in Figure 4. 


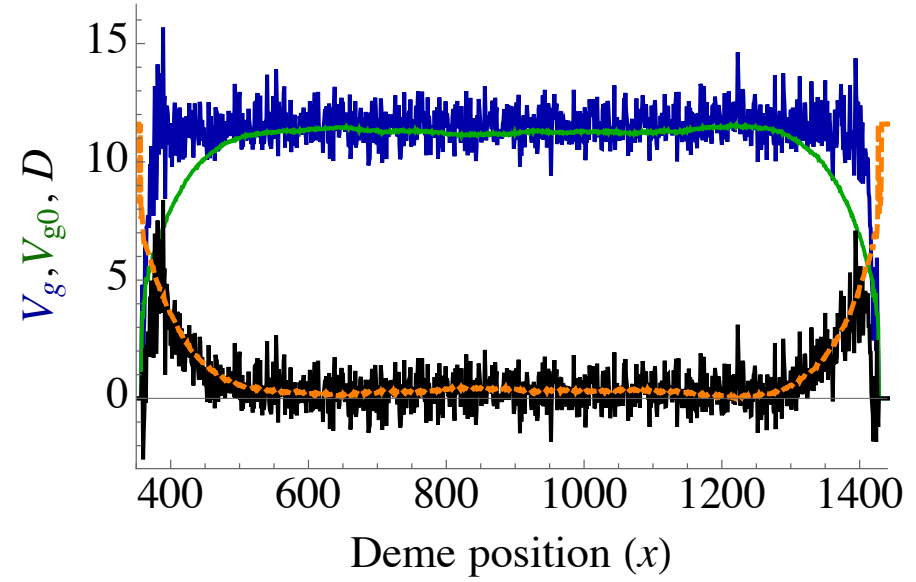

Figure S5. Genetic variance $V_{\mathrm{g}}$ (blue), genic variance $V_{\mathrm{g}, 0}$ (green) and contribution of linkage disequilibria to the genetic variance $D=V_{\mathrm{g}}-V_{\mathrm{g}, 0}$ (black) along a sexual metapopulation with $s=0.001, b=1, V_{\mathrm{s}}=20, \sigma=4, K_{\max }=500, \omega=100$, $r_{\mathrm{m}}=1.1$ and $U=10^{-3}$. Orange curve: predicted value of $D$ obtained from the theoretical prediction for $V_{\mathrm{g}}$ (equation 6) and the observed $V_{\mathrm{g}, 0} \cdot V_{\mathrm{g}, 0}$ decreases at the range margins while $D$ increases, leading to an approximately constant $V_{\mathrm{g}}$. 

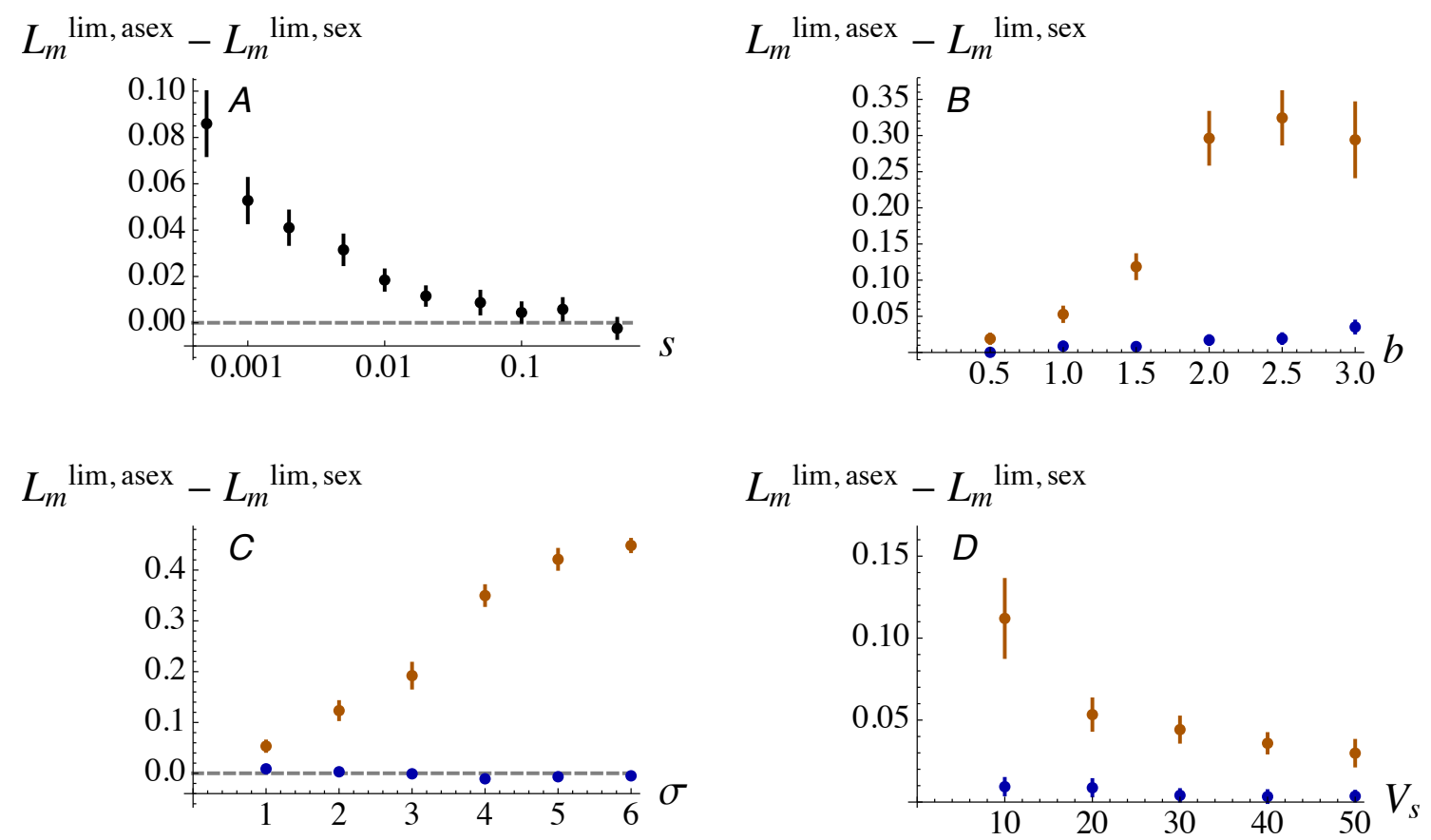

Figure S6. Difference in migration load $L_{\mathrm{m}}$ between asexual and sexual populations at equilibrium, in the margins of the distribution range, as a function of $s, b, \sigma$ and $V_{\mathrm{s}}$ (default parameter values are as in Figures 3 and 5; blue: $s=0.05$, brown: $s=0.001$ ). The migration load is averaged over the demes located at both edges of the range, in which deme size is comprised between 10 and 50 individuals. 

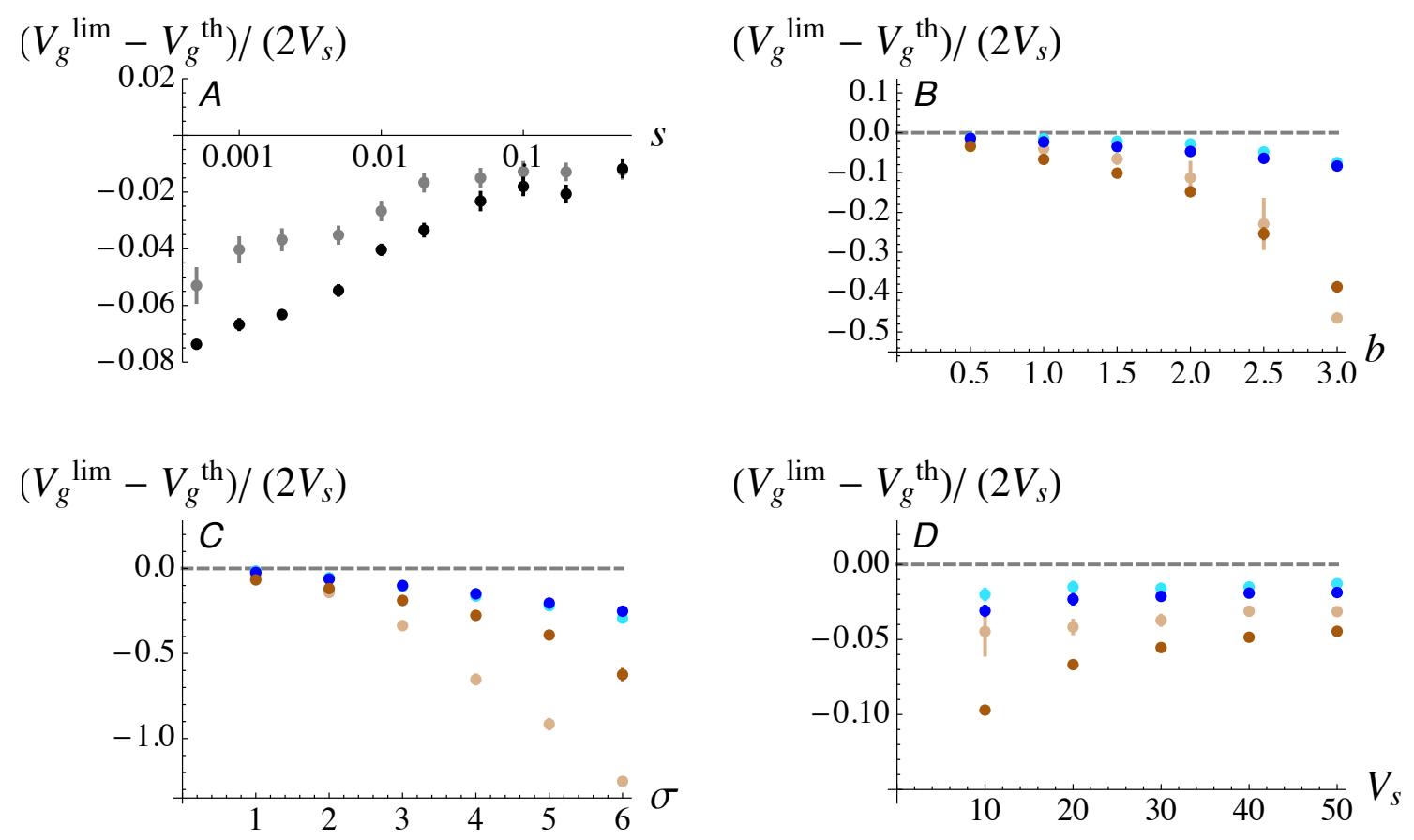

Figure S7. Difference between the average genetic variance $V_{g}$ per deme observed in the simulations and the theoretical prediction given by equation $6\left(V_{g}^{\text {th }}\right)$, scaled by $2 V_{\mathrm{s}}$, in sexual and asexual populations, and at the edges of the distribution range of the population (values are averaged over demes in which population size is comprised between 10 and 50 individuals). Parameter values are as in Figures 3, 5 and 6, colors have the same meaning as in Figure 6. 


$$
\begin{aligned}
& \text { range }^{\text {asex }}-\text { range }^{\text {sex }}
\end{aligned}
$$

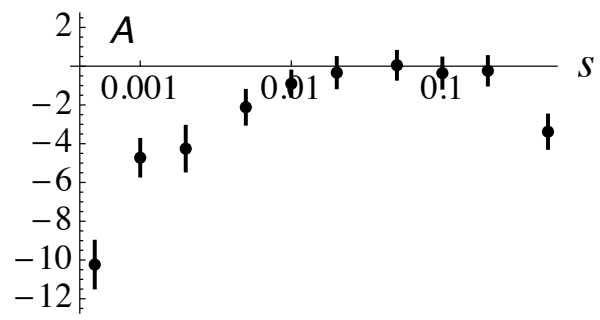
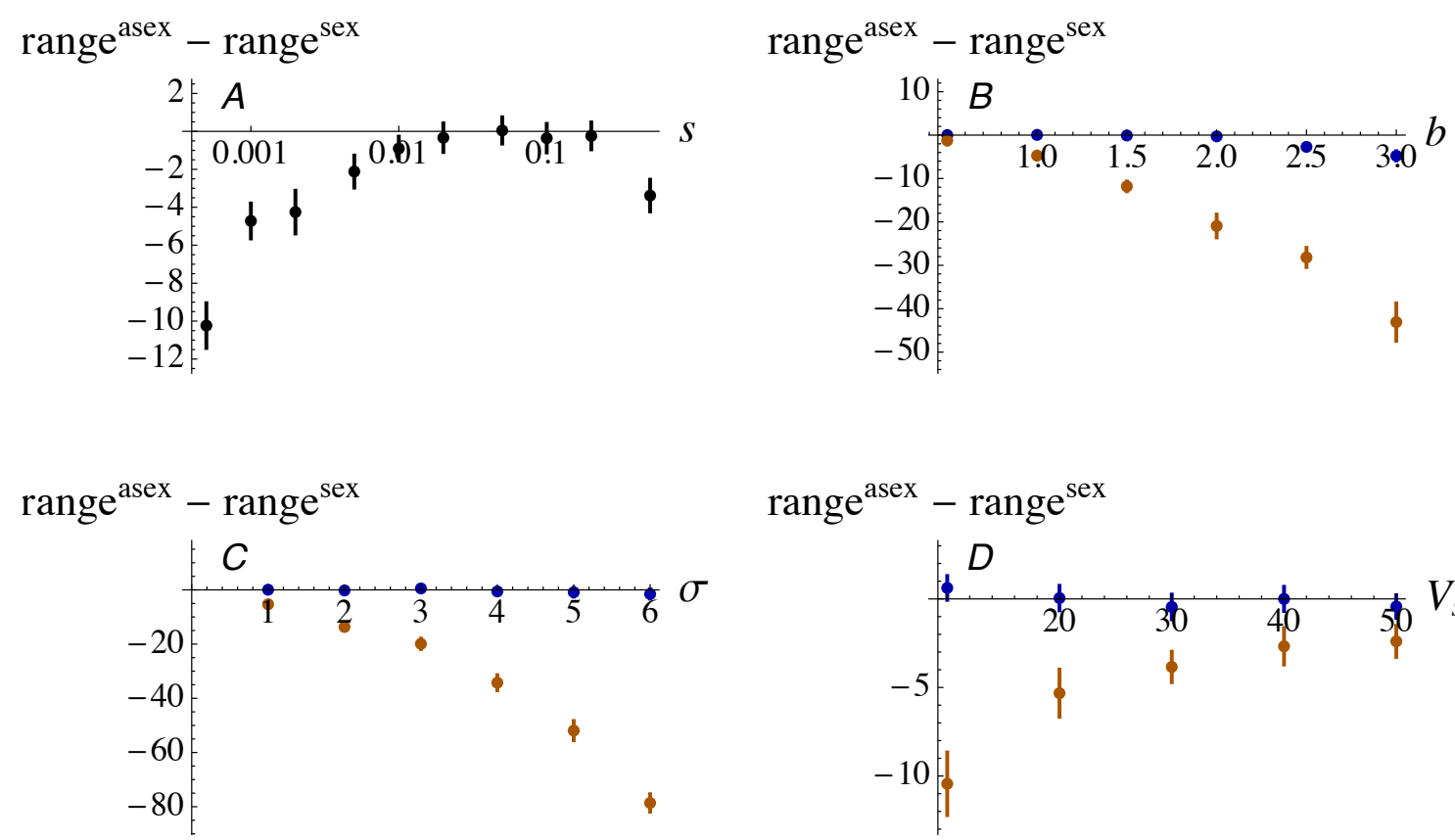

range $^{\text {asex }}-$ range $^{\text {sex }}$

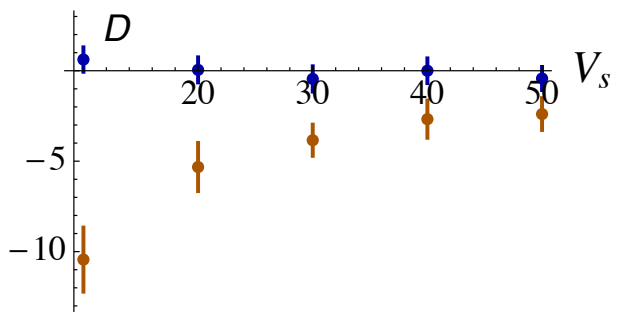

Figure S8. Difference in the range size of asexual and sexual populations at equilibrium (defined as the length of the area over which deme size is higher than 10 individuals), for the same parameter values as in Figures 3,5 and 6. 

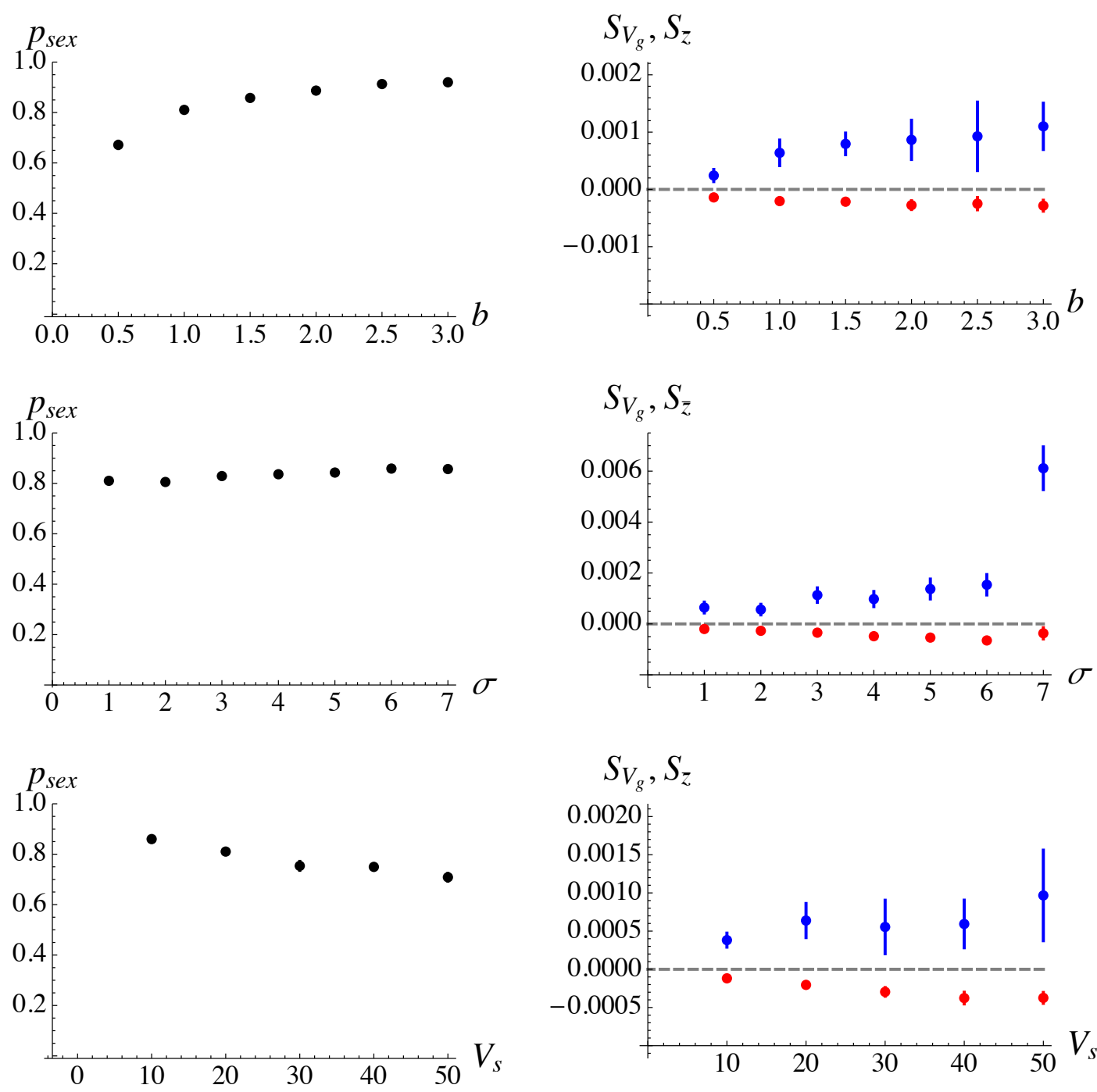

Figure S9. Left: mean frequency of sexuals at equilibrium in the central part of the distribution range as a function of $b, \sigma$ and $V_{\mathrm{s}}$, for the same default parameter values as in Figure 3, and $c=1$ (no cost of sex). Right: the two components of selection for sex $S_{V_{\mathrm{g}}}$ (blue) and $S_{\bar{z}}$ (red), given by equations 4 and 5 . 


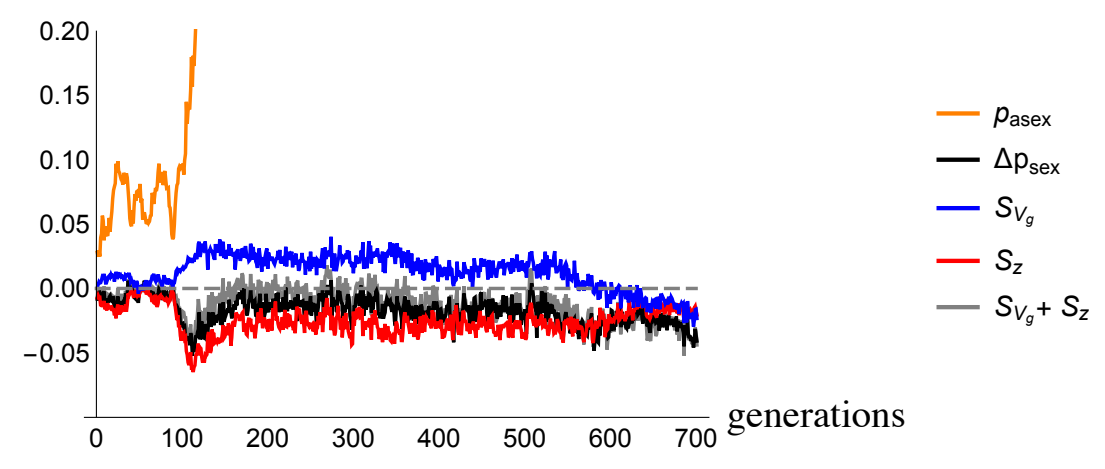

Figure S10. The spread of asexuals at range limits is driven by $S_{\bar{z}}$ : the orange curve shows the frequency of asexuals over time (after the preliminary generations) averaged over 20 demes at one of the range limits, while the blue and red curves show the two components of selection for sex $S_{V_{\mathrm{g}}}$ (blue) and $S_{\bar{z}}$ (red). The black curve shows the observed change in frequency of sexuals per generation, while the grey curve shows $S_{V_{\mathrm{g}}}+S_{\bar{z}}$. Parameter values are as in Figure 3, with $\sigma=4$. 

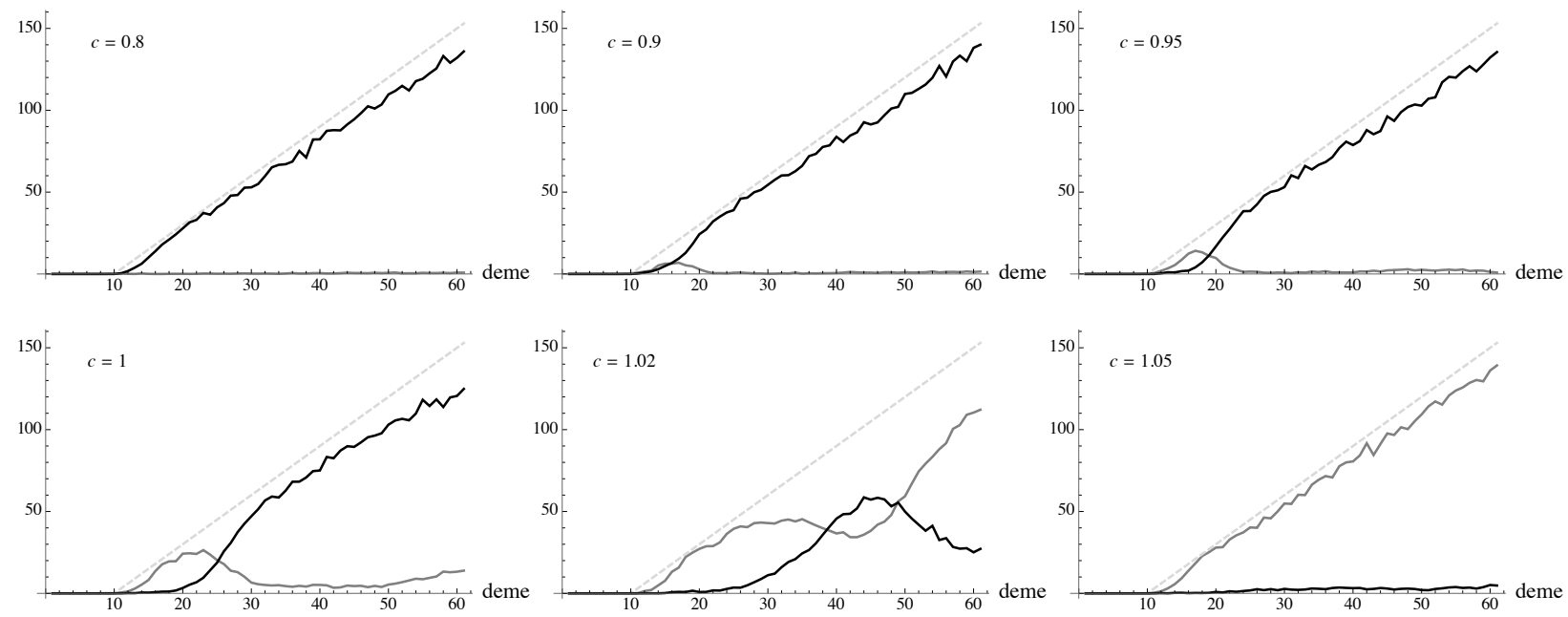

Figure S11. Number of sexual (black) and asexual (grey) individuals per deme at the left limit of the population range (averaged over the last 20,000 generations of the simulation), for different values of the cost of $\operatorname{sex} c$ (note that $c<1$ generates an intrinsic advantage for sexuals). Default parameter values are as in Figure 3 with $s=0.001$. The carrying capacity $K$ is shown in dotted grey. 

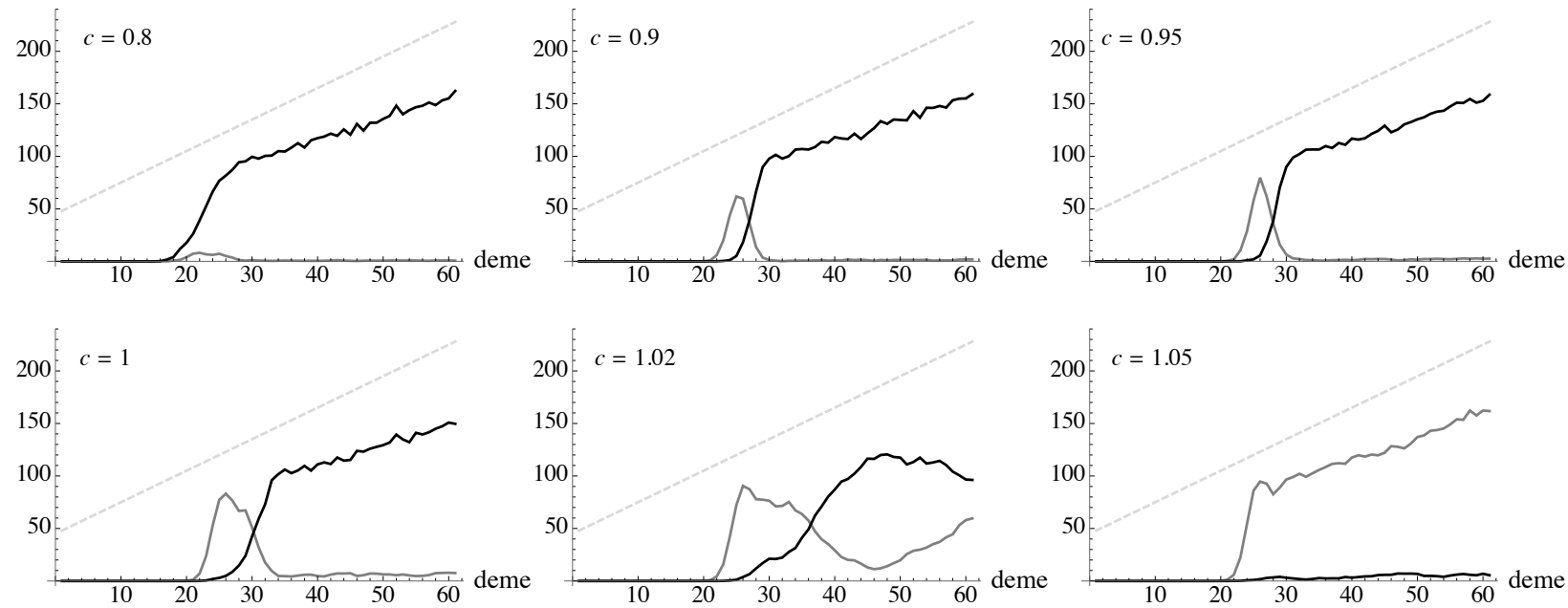

Figure S12. Number of sexual (black) and asexual (grey) individuals per deme at the left limit of the population range: same as Figure S11 with $V_{\mathrm{s}}=2$ (stronger selection on the trait). 

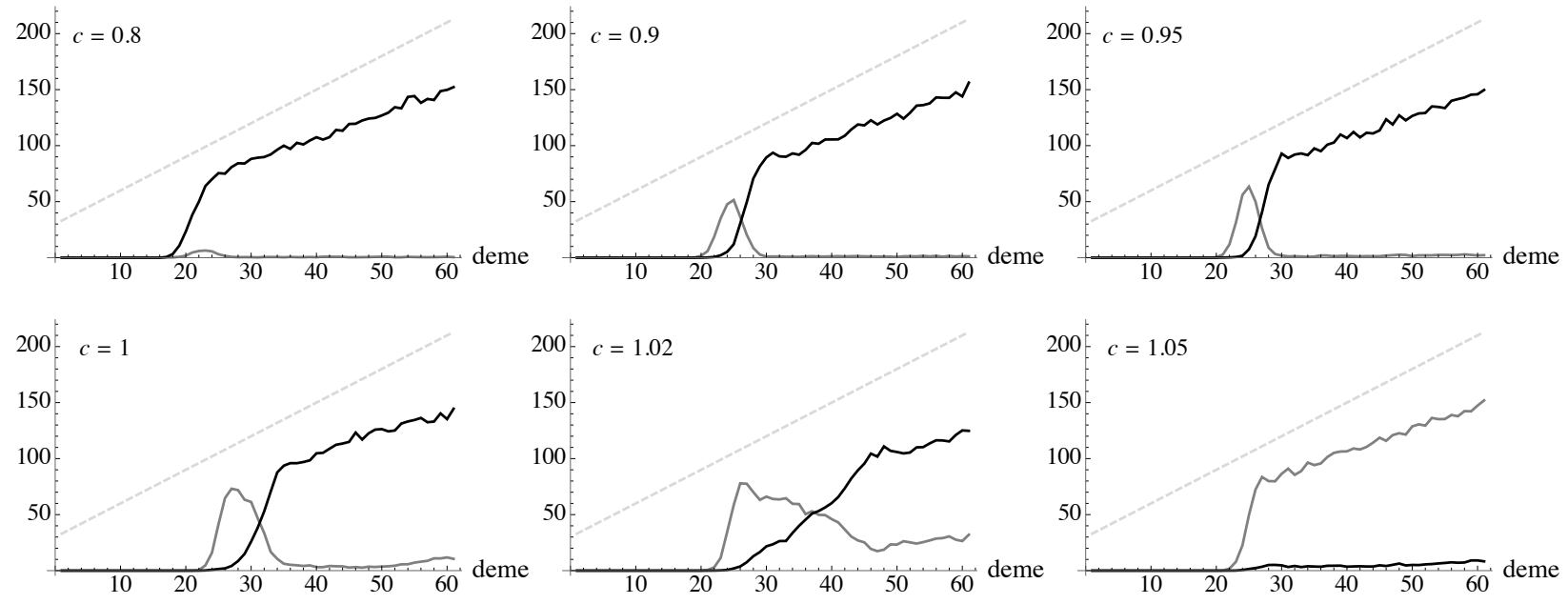

Figure S13. Number of sexual (black) and asexual (grey) individuals per deme at the left limit of the population range: same as Figure S11 with $b=3$ (stronger environmental gradient). 

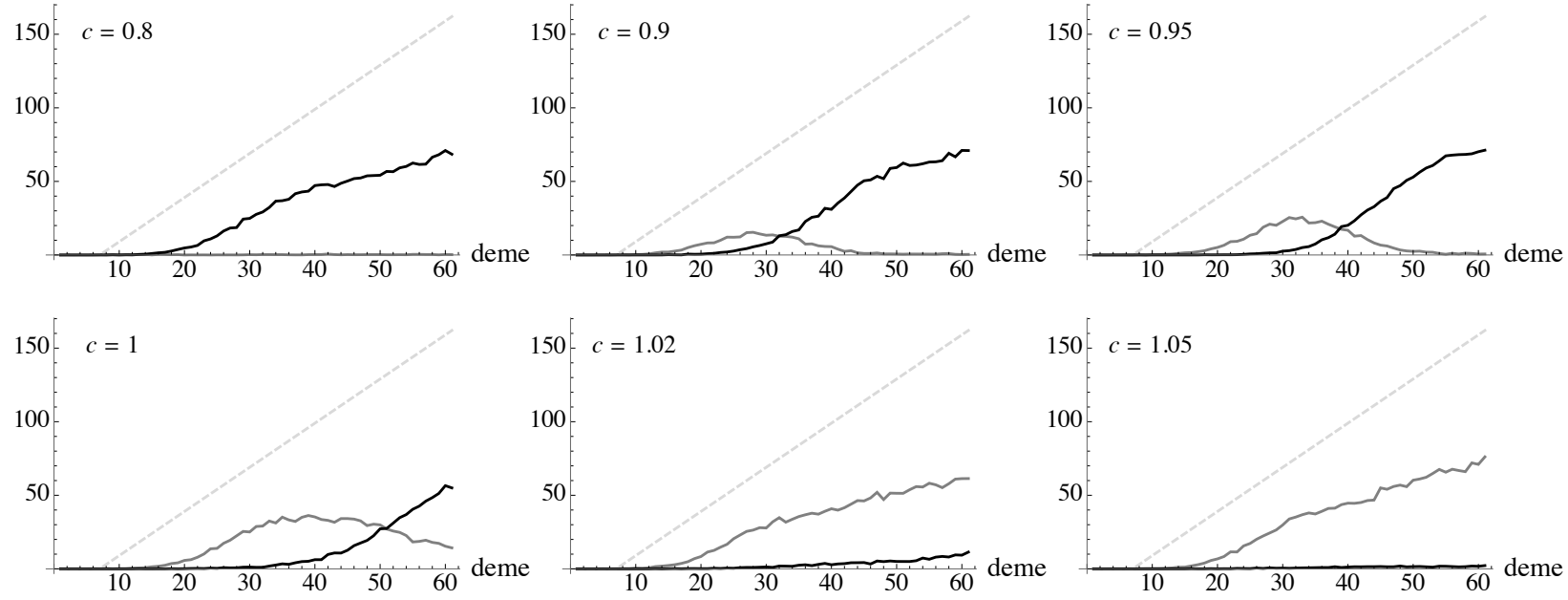

Figure S14. Number of sexual (black) and asexual (grey) individuals per deme at the left limit of the population range: same as Figure S11 with $\sigma=6$ (higher dispersal distance). 

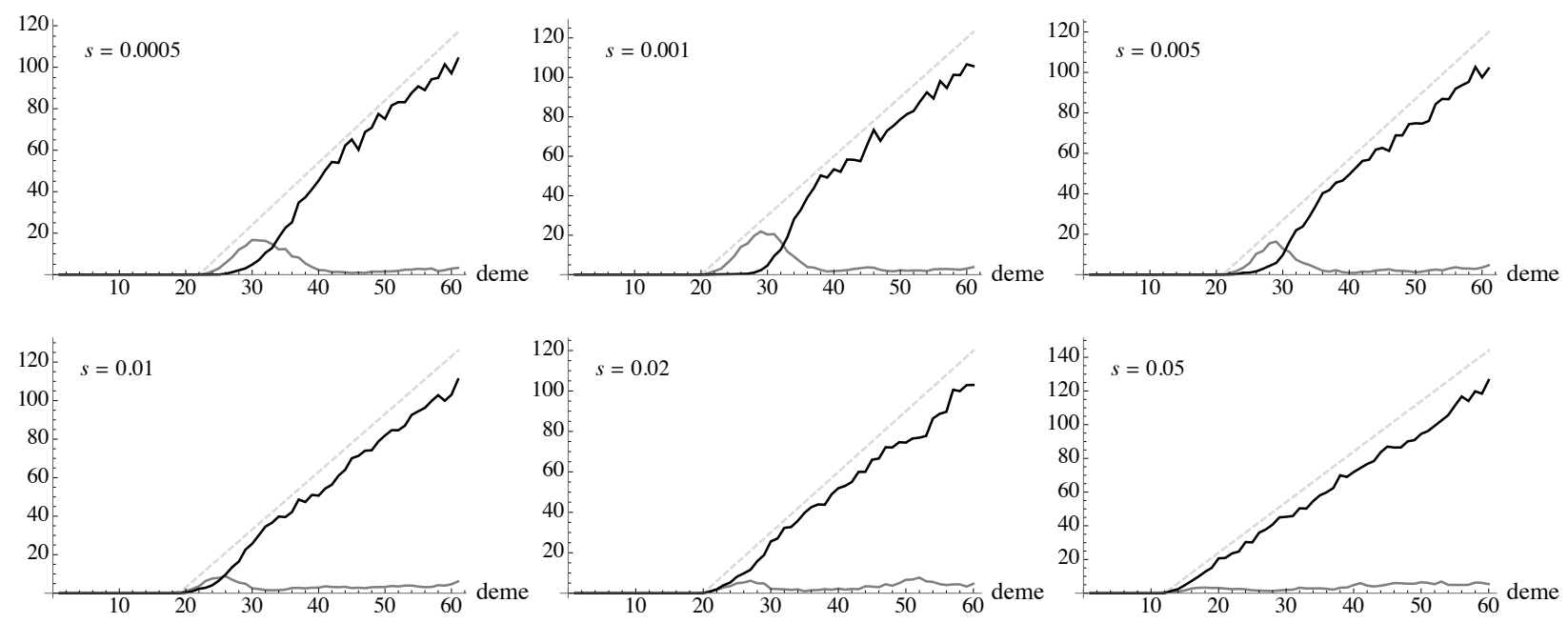

Figure S15. Number of sexual (black) and asexual (grey) individuals per deme at the left limit of the population range, for $c=0.98$ and different values of $s$ (strength of selection at loci coding for the trait). Default parameter values are as in Figure 3. 

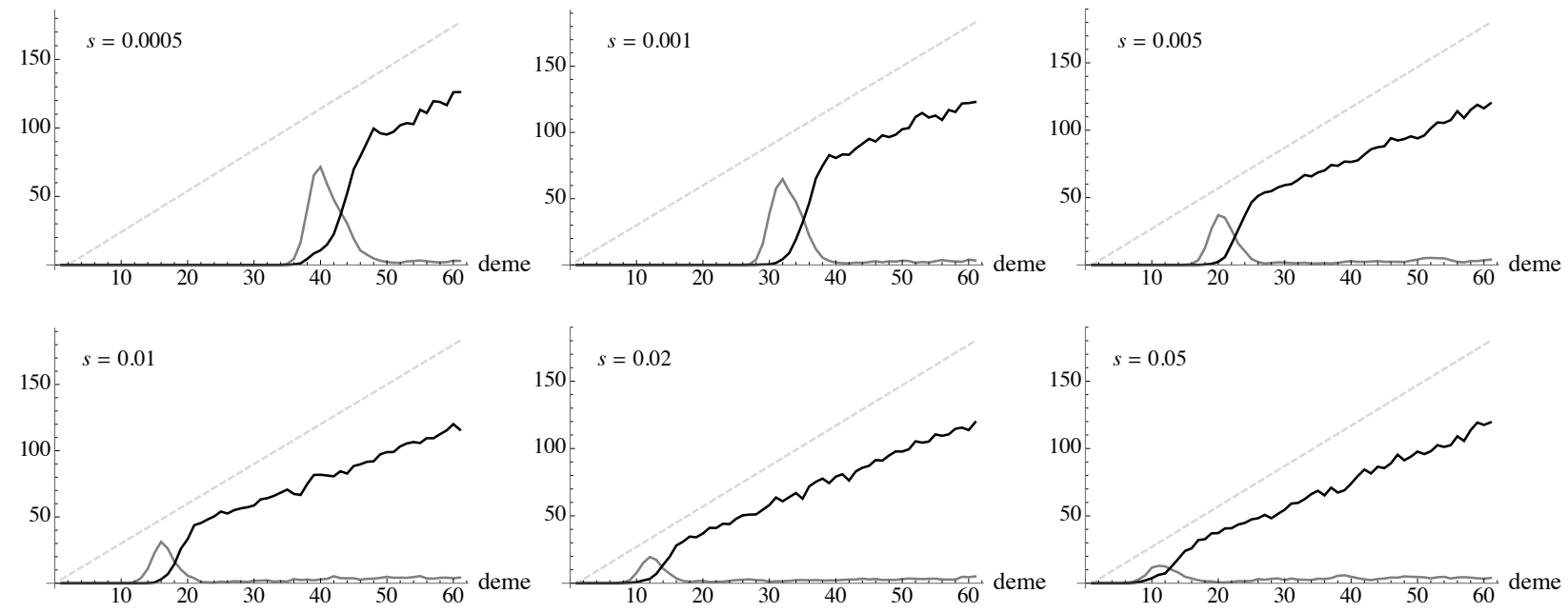

Figure S16. Same as Figure S15 for $V_{\mathrm{s}}=2$ (stronger selection on the trait). 